\title{
Maximal Overlap with the Fully Separable State and Translational Invariance for Multipartite Entangled States
}

\author{
H. T. Cui(崔海涛)*, Di Yuan(袁地) and J. L. Tian(田俊龙) \\ School of Physics and Electrical Engineering, Anyang Normal University, Anyang 455000, China
}

(Dated: May 30, 2018)

\begin{abstract}
The maximal overlap with the fully separable state for the multipartite entangled pure state with translational invariance is studied explicitly by some exact and numerical evaluations, focusing on the one-dimensional qubit system and some representative types of translational invariance. The results show that the translational invariance of the multipartite state could have an intrinsic effect on the determinations of the maximal overlap and the nearest fully separable state for multipartite entangled states. Furthermore a hierarchy of the basic entangled states with translational invariance is founded, from which one could readily find the maximal overlap and a related fully separable state for the multipartite state composed of different translational invariance structures.
\end{abstract}

PACS numbers: 03.65.Ud

\section{INTRODUCTION}

Quantum entanglement is considered as the most distinct feature in the quantum world to the classical world. Generally it is the manifestation of the nonlocal connectedness in the distinguishable parties, where the nonlocal means that if only this connectedness is constructed, it does not disappear automatically until the appearance of decoherence or local measurements, even if the parties are space-like separated from each other. An important consequence of this connectedness is that the behaviors of any one party are inevitably affected by the other parties. Thus it is convenient for the quantification of the connectedness to find the reduced density matrix of the subsystem. Some successful criteria or measures of quantum entanglement have been proposed along this line (see Ref [1] for a comprehensive review).

It should be pointed out that the intuition of quantum entanglement is mainly from the understanding of Bell states, which are maximally entangled states of two-qubit systems. Particularly because Bell states are bipartite, one can obtain complete information on the entanglement from the reduced density matrix of the subsystem. However the situation becomes complex when extended to multipartite systems. Multipartite entanglement could exist when the multipartite state cannot be written as the fully separable form

$$
\rho_{f}^{s e p}=\sum_{i} p_{i} \rho_{1}^{(i)} \otimes \rho_{2}^{(i)} \otimes \cdots \rho_{N}^{(i)}
$$

where $N$ is the number of the distinguishable party, and $p_{i}$ denotes the joint probability distribution of the single-party state $\rho_{n}^{(i)}(n=1,2, \cdots, N)$. In contrast to the bipartite entanglement defined as the violation of the bi-separable form for the bipartite state $\rho_{b}^{s e p}=$ $\sum_{i} p_{i} \rho_{1}^{(i)} \otimes \rho_{2}^{(i)}$, there exists the multi-connectedness in

*Electric address: cuiht@aynu.edu.cn multipartite states, which is hard to characterize completely by the reduced density matrix of the subsystem only.

This distinction between bipartite and multipartite entanglement has led to several intrinsic observations. It is known that there exist two inequivalent threequbit entangled states, the $W$ state and GreenbergerHorn-Zeilinger (GHZ) state, which are not interconvertible under local operations and classical communications (LOCCs) [2]. Furthermore, a limit to the distribution of entanglement in multipartite states is found first for the three-qubit case [3], and then for the arbitrary multipartite case [4]. These phenomena imply that one need some special methods to describe the connectedness embedded in multipartite states.

The global approach is a natural choice to obtain the comprehensive information of the connectedness in multipartite states. In contrast to the dependence of the measurements of bipartite entanglement on the subsystem, the global measurement of multipartite entanglement instead focuses mainly on the overall state instead. For instance the relative entropy of entanglement is one type of measurement, defined as [5]

$$
E_{R}=\min _{\left\{\rho^{s e p}\right\}} \operatorname{Tr}\left[\rho\left(\log \rho-\log \rho^{s e p}\right)\right] .
$$

The key idea in this measurement is that the closer to the separable states is $\rho^{s e p}$, the less entangled is $\rho$. Global robustness is another global measure of entanglement, of which the main idea is to quantify how robust is the entangled state against the environmental noise [6]. In addition there exists a global criterion of entanglement: entanglement witness. The idea is to find a special Hermitian operator, whose expectation value with the multipartite state is positive or zero when this state is fully separable, while it is negative when this state is entangled 7].

Similar to the relative entropy of entanglement, geometric entanglement (GE) is another global measure of entanglement, related directly to the distance between the entangled state and the fully separable state in 
Hilbert space. GE is defined generally for the pure state as [8]

$$
E_{g}=\min _{\{|\phi\rangle\}} \||\psi\rangle-|\phi\rangle \|^{2},
$$

where $\|\cdots\|$ denotes the norm, or equivalently

$$
E_{g}=1-\Lambda_{\max }^{2}=1-\max _{\{|\phi\rangle\}}|\langle\psi \mid \phi\rangle|^{2} .
$$

where $|\phi\rangle=\otimes_{i=1}^{N}\left|\phi^{i}\right\rangle$ is a fully separable pure state, $\left|\phi^{i}\right\rangle$ denotes the single-party state, and $\Lambda_{\max }$ denotes the maximal overlap of $|\psi\rangle$ and $|\phi\rangle . E_{g}$ is determined geometrically by the overlap angle between the state vectors $|\psi\rangle$ and $|\phi\rangle$ in Hilbert space. Thus the optimal in the definitions above can be reduced to find the nearest fully separable state $|\phi\rangle$, which has a minimal overlap angle with an entangled state $|\psi\rangle$. Furthermore, the determination of the nearest $|\phi\rangle$ is equivalent physically to the determination of the Hartree-Fock approximation ground state of the auxiliary Hamiltonian $H=-|\psi\rangle\langle\psi|$ [8]. Additionally our recent study shows that $\Lambda_{\max }$ would be a direct generalization of the concept of Anderson orthogonality catastrophe (AOC) in solid theory [9], and thus GE can be used to also describe the correlations in manybody systems [10 12]. This point can be manifested by the expression

$$
\Delta=\left|\left\langle\Phi \mid \Phi^{p}\right\rangle\right|^{2},
$$

where $|\Phi\rangle$ corresponds to the true ground state of a manybody system, and $\left|\Phi^{p}\right\rangle$ is actually a pure product state described entirely in terms of free plane waves, which can be considered as the ground state without potential [13]. AOC denotes the vanishing of $\Delta$ under a thermodynamic limit, even for a very weak potential. This feature discloses that the correlation in many-body systems is the intrinsic character that is different from the free system without potential. Then, by finding the tendency of $\Delta$ under thermodynamic limit, one can obtain information on the correlation in systems. With these points, GE actually defines a measurement of the correlation in multipartite states, independent of the details of the system because of the optimal choice of the fully separable state. Furthermore because of the optimal the measured correlation is evidently quantum, as proved in the next section.

These distinct characters display the popularity of GE as an description of the connectedness in multipartite states. Also GE has become one of the most accepted measures of multipartite entanglement. However, it is difficult in general to find the maximal overlap $\Lambda_{\max }$ because of the optimal of $|\phi\rangle$, which is the crucial point for the evaluation of GE, and there are few exact results [8, 11, 12, 14 22. Recently important progress has been made for the entangled state with permutational invariante that the nearest fully separable state for it is necessarily permutational invariance [16 18]; that is to say, it is always an optimal choice to set $|\phi\rangle=\left|\phi^{\prime}\right\rangle^{\otimes N}$ in order to find $\Lambda_{\max }$ of this type of entangled state, in which $\left|\phi^{\prime}\right\rangle$ denotes the single-party state. This important conclusion implies strongly that it would reduce the optimal determination of GE by utilizing the symmetry of $|\psi\rangle$. Furthermore our recent study also shows that the maximal overlap could be obtained if the fully separable state, either pure or mixed, shows the same global symmetry to the entangled state [9], which means, mathematically, that the two states should belong to the same symmetric subspace.

However, except for permutationally invariant entangled states, there are few examples for the evaluations of GE for other multipartite states. This article serves to fill this gap partially. For this purpose, GE for the multipartite states with translational invariance is studied explicitly through some exact and numerical examples, which are focused on the qubit system with the geometry of circles and some representative types of translational invariant entangled states. Another reason for this choice comes from revisiting the current points of the determination of $\Lambda_{\max }$ for translationally invariant states, appearing in some very recent works; for examples, one case is that the nearest fully separable state could not be determined by the translational invariance of the entangled state, and thus this symmetry would be helpless for the reduction of the optimal in GE [17]. One case is to set $|\phi\rangle=\left|\phi^{\prime}\right\rangle^{\otimes N}$ in order to obtain $\Lambda_{\max }$ of the ground state in translationally invariant many-body systems [8, 21]. Another case is to adopt the maximal coefficient under the product state basis as the maximal overlap [15], etc.

Through several exact and numerical calculations, we try to illustrate convincingly in this article that the optimal determination of the nearest fully separable state and maximal overlap with a fully separable state for translationally invariant entangled states can be reduced greatly by utilizing the translational invariance of the entangled state. Additionally, the above points can occurs only for some special cases. Furthermore our study shows that there exists a hierarchy for the so-called basic translationally invariant entangled states defined in Sec $\mathrm{V}$ from which one can decide directly the nearest fully separable state and the $\Lambda_{\max }$.

\section{TECHNICAL PREPARATIONS}

Some concepts are clarified in this section. At the end of this section we present a proof for the point that for an entangled pure state there always exists a nearest fully separable pure state.

Permutational invariance (PI) denotes formally the situation that the multipartite state is unchanged by exchanging the states of two arbitrary single parties. For example, the $N$-qubit GHZ state,

$$
|\mathrm{GHZ}\rangle_{N}=\frac{1}{\sqrt{2}}(|11 \cdots 1\rangle+|00 \cdots 0\rangle)
$$

is obviously permutationally invariant because all parties always have the same state simultaneously. In contrast, 


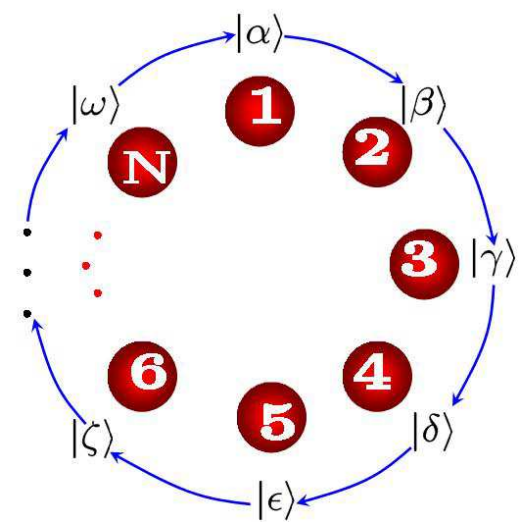

FIG. 1: A schema for the TI of multipartite state, in which the numbers refer to different parties and the greek letters denote the single-party states. The arrows represent the cyclic translation of all single-party states.

the generalized $W$ state,

$$
|W\rangle_{N}=\frac{1}{\sqrt{N}}(|10 \cdots 0\rangle+|010 \cdots 0\rangle+\cdots+|0 \cdots 01\rangle)
$$

is slightly special; although it is also permutationally invariant, the key feature is that $|W\rangle_{N}$ includes all possible combinations of the single $|1\rangle$ and the $(N-1)$ 's $|0\rangle$. This difference would induce a distinct hierarchy from $|\mathrm{GHZ}\rangle_{N}$, as shown in Sec $\nabla$ Similar to the $|W\rangle_{N}$ state, the Dicke state has the same feature, which is defined as

$$
|S(N ; n)\rangle=\sqrt{\frac{n !(N-n) !}{N !}} \sum_{\text {permutation }}|\underbrace{0 \cdots 0}_{n} \underbrace{1 \cdots 1}_{N-n}\rangle .
$$

Translational invariance (TI) denotes the situation where the multipartite state is unchanged under the cyclic translation of the single-party states. It should pointed out that this definition is different from the TI defined in physical space, e.g. in lattice systems, for which TI depends heavily on the geometry of the physical space. Actually because we are only interested in the connectedness of states belonging to distinguishable parties, it is unimportant in this case as to how one may realize this multipartite state in realistic physical systems. The crucial point for the TI of the multipartite state is that any party could be distinguished from the others by a definite label, and the single-party state could belong to any party by a cyclic translation, as show in Fig. 1] Admittedly, it is, in general, related to the geometry of the physical space as to how one should label all parties. However, a sequence of all parties can always be constructed only if the labels are definite. Our definition of TI of multipartite states is simply based on this sequence, and thus is indirect to the geometry of systems. In fact, the multipartite states studied in this article can be considered with the geometry of the circle, as shown in Fig. 1.

An example is the state

$$
\left|\mathrm{GHZ}^{\prime}\right\rangle_{N}=\frac{1}{\sqrt{2}}(|1010 \cdots 10\rangle+|0101 \cdots 01\rangle)
$$

which can be obtained by imposing the local unitary operation $\sigma_{2}^{x} \otimes \sigma_{4}^{x} \otimes \cdots \otimes \sigma_{2 n}^{x} \otimes \cdots$ on $|\mathrm{GHZ}\rangle_{N}$. The key feature of $\left|\mathrm{GHZ}^{\prime}\right\rangle_{N}$ is the invariance by translating all single-party states cyclicly. Interestingly, there is a cyclic structure for $\left|\mathrm{GHZ}^{\prime}\right\rangle_{N}$ where "1" and "0" appear periodically at the next-nearest-neighbor site [23]. Furthermore this structure also determines the times of cyclic translation in order to span all terms in the multipartite state. For $\left|\psi^{2}\right\rangle_{4}=\frac{1}{2}(|1100\rangle+|0110\rangle+|0011\rangle+|1001\rangle)$ as a general example, there is no periodic structure similar to $\left|\mathrm{GHZ}^{\prime}\right\rangle_{N}$, and it has to include all possibilities after cyclic translation for an arbitrary term in $\left|\psi^{2}\right\rangle_{4}$ in order to maintain TI. There is also a hierarchy for the multipartite states with TI because of the different cyclic structures. Further discussions will be presented in Secs. III and V A trivial observation is that PI also means TI, and so the discussion below will not distinguish between them if it is not necessary.

An important question is what should be the form of the nearest fully separable state for an entangled pure state, which decides the procedure adopted to find $\Lambda_{\max }$. The general method is to suppose that the nearest fully separable state is still pure [8], however that has never been proved exactly to the best of our knowledge. We present a proof for this point here.

Proof: Consider the fully separable state defined in Eq.(11) and an entangled pure state $|\psi\rangle$. Then the overlap is written as

$$
\begin{aligned}
\operatorname{Tr}\left[\rho_{f}^{s e p}|\psi\rangle\langle\psi|\right] & =\sum_{i} p_{i} \operatorname{Tr}\left[\rho_{1}^{(i)} \otimes \rho_{2}^{(i)} \otimes \cdots \rho_{N}^{(i)}|\psi\rangle\langle\psi|\right] \\
& =\sum_{i} p_{i} \Delta_{i} .
\end{aligned}
$$

Let $\Delta_{1} \geq \Delta_{2} \geq \cdots$, and one has inequality $\operatorname{Tr}\left[\rho_{f}^{s e p}|\psi\rangle\langle\psi|\right] \leq \Delta_{1}$. Thus

$$
\max _{\left\{\rho_{f}^{s e p}\right\}}\left\{\operatorname{Tr}\left[\rho_{f}^{s e p}|\psi\rangle\langle\psi|\right]\right\} \Leftrightarrow \max _{\left\{\rho^{s}\right\}} \operatorname{Tr}\left[\rho_{1} \otimes \rho_{2} \otimes \cdots \otimes \rho_{N}|\psi\rangle\langle\psi|\right],
$$

where $\rho^{s}=\rho_{1} \otimes \rho_{2} \otimes \cdots \otimes \rho_{N}$, and $\Leftrightarrow$ means "equivalent".

The crucial observation is that $\rho^{s}$ can be rewritten under the product state basis as

$$
\rho^{s}=\sum_{i} \varrho_{i}\left|\phi_{i}\right\rangle\left\langle\phi_{i}\right|,
$$

where the set of fully separable pure states $\left|\phi_{i}\right\rangle=$ $\otimes_{n=1}^{N}\left|\phi_{n}^{(i)}\right\rangle$ constitutes a product state basis. Then

$$
\operatorname{Tr}\left[\rho^{s}|\psi\rangle\langle\psi|\right]=\sum_{i} \varrho_{i}\left|\left\langle\psi \mid \phi_{i}\right\rangle\right|^{2}=\sum_{i} \varrho_{i} \delta_{i} .
$$

Let $\delta_{1} \geq \delta_{2} \geq \cdots$, and then the overlap satisfies the inequality

$$
\operatorname{Tr}\left[\rho^{s}|\psi\rangle\langle\psi|\right] \leq \delta_{1} .
$$

This important result means

$$
\max _{\left\{\rho^{s}\right\}} \operatorname{Tr}\left[\rho^{s}|\psi\rangle\langle\psi|\right] \Leftrightarrow \max _{\{|\phi\rangle\}}|\langle\psi \mid \phi\rangle|^{2} .
$$


One reaches the final conclusion that the determination of the maximal overlap with the nearest fully separable state for an entangled pure state is equivalent to that with a fully separable pure state, i.e.,

$$
\max _{\left\{\rho_{f}^{s e p}\right\}}\left\{\operatorname{Tr}\left[\rho_{f}^{s e p}|\psi\rangle\langle\psi|\right]\right\} \Leftrightarrow \max _{\{|\phi\rangle\}}|\langle\psi \mid \phi\rangle|^{2}
$$

This relation shows that the nearest fully separable state for an entangled pure state is only necessarily pure because some inequalities appear in this proof. Respecting that $\rho_{f}^{s e p}$ contains only the classical correlation [24], GE actually measures the minimal distance to the classical state in Hilbert space by an optimal choice of $\rho_{f}^{s e p}$, and thus quantifies the nonclassical correlation in the entangled state $|\psi\rangle$.

An interesting case is that the maximal $\delta_{i}$ is not unique, and then the nearest fully separable state may be mixed. A typical example is the determination of the nearest fully separable state for $\left|\mathrm{GHZ}^{\prime}\right\rangle_{N}$, which is known as the pure state $|1010 \cdots 10\rangle$ or $|0101 \cdots 01\rangle$ (see Sec. IIIF. However, one can check easily that $\Lambda_{\max }$ has the same value for $\frac{1}{2}(|1010 \cdots 10\rangle\langle 1010 \cdots 10|+$ $|0101 \cdots 01\rangle\langle 0101 \cdots 01|)$. Thus we claim that it is enough for the determination of the maximal overlap $\Lambda_{\max }$ for an entangled pure state to focus only on the fully separable pure state. Thus, in the following sections, we adopt the notation

$$
\begin{aligned}
& \left|\phi\left(a_{1}, a_{2}, \cdots, a_{N} ; \theta_{1}, \theta_{2}, \cdots, \theta_{N}\right)\right\rangle \\
= & \otimes_{i=1}^{N}\left(\sqrt{a_{i}}|1\rangle_{i}+\mathrm{e}^{i \theta_{i}} \sqrt{1-a_{i}}|0\rangle_{i}\right)
\end{aligned}
$$

where $a_{i} \in[0,1]$ and $\theta_{i} \in[0,2 \pi)$ denote the $N$-qubit fully separable pure state (FSPs).

\section{FINDING THE MAXIMAL OVERLAP I: ROLE OF TRANSLATIONAL INVARIANCE}

With these preparations, we are ready to evaluate $\Lambda_{\max }$ for translationally invariant entangled states. As shown by the exact evaluations below, there always exists the nearest fully separable state with the same TI to entangled state, which can be constructed by the equiprobably incoherent superposition of the FSPs with the same $\Lambda_{\max }$. This result means that one can reduce the optimal of $\Lambda_{\max }$ by utilizing the TI of the entangled state. For this purpose, the discussion in this section is implemented mainly for the so-called basic TI entangled states with the geometry of circle, where the meaning of basic is that $|\psi\rangle$ is composed of only one type of cyclic structure (the hybrid case will be explored in Sec. V]). Although there is no exact proof, some exact or numerical examples are presented instead in order to demonstrate the validity of our points.

\section{A. 3-qubit case}

It is known that there are two inequivalent multipartite entangled states in this case: GHZ and $W$ states. Although their GEs have been studied extensively, the calculation of their $\Lambda_{\max }$ here is to show the general methods for the evaluation of $\Lambda_{\max }$, adopted in this section.

1. $|\mathrm{GHZ}\rangle=\frac{1}{\sqrt{2}}(|111\rangle+|000\rangle)$

The overlap with $|\phi\rangle$ is written as

$$
\begin{aligned}
& |\langle\mathrm{GHZ} \mid \phi\rangle|^{2}= \\
& \left.\frac{1}{2}\left|\sqrt{a_{1} a_{2} a_{3}}+\mathrm{e}^{i\left(\theta_{1}+\theta_{2}+\theta_{3}\right)} \sqrt{\left(1-a_{1}\right)\left(1-a_{2}\right)\left(1-a_{3}\right)} t^{2}\right\rangle\right)
\end{aligned}
$$

With the inequality

$$
x_{1}^{n}+x_{2}^{n}+\cdots+x_{N}^{n} \geq N\left(x_{1} x_{2} \cdots x_{N}\right)^{\frac{n}{N}}
$$

where the equality happens if and only if (iff) $x_{1}=x_{2}=$ $\cdots=x_{N}$, we have

$$
\begin{aligned}
|\langle\mathrm{GHZ} \mid \phi\rangle|^{2} & \leq \frac{1}{2}\left|a^{\frac{3}{2}}+\mathrm{e}^{i\left(\theta_{1}+\theta_{2}+\theta_{3}\right)}(1-a)^{\frac{3}{2}}\right|^{2} \\
& \leq \frac{1}{2}\left[a^{\frac{3}{2}}+(1-a)^{\frac{3}{2}}\right]^{2}
\end{aligned}
$$

where the first equality happens iff $a_{1}=a_{2}=a_{3}=a$, and the second only if $\theta_{1}+\theta_{2}+\theta_{3}=2 m \pi$ ( $m$ is an integer). Consequently the optimal for six independent variables becomes for a single one, $a$, i.e.,

$$
\max _{|\phi\rangle}|\langle\mathrm{GHZ} \mid \phi\rangle|^{2} \Leftrightarrow \frac{1}{2} \max _{a}\left[a^{\frac{3}{2}}+(1-a)^{\frac{3}{2}}\right]^{2},
$$

which can be shown easily that its maximal values is $\frac{1}{2}$ when $a=1$ or $a=0$.

Then the nearest fully separable state can be chosen as $|111\rangle$ or $|000\rangle$, or their incoherent superposition with equal amplitude $\frac{1}{2}(|111\rangle\langle 111|+| 000\rangle\langle 000|)$. This observation shows that there always exists the fully separable state with PI by choosing $\theta_{1}=\theta_{2}=\theta_{3}$, whether it is pure or mixed. With respect to the limit $\theta_{1}+\theta_{2}+\theta_{3}=2 m \pi$, this result is only necessary.

$$
\text { 2. }|W\rangle=\frac{1}{\sqrt{3}}(|100\rangle+|010\rangle+|001\rangle)
$$

By the method discussed in Appendix A one can easily obtain $\Lambda_{\max }=4 / 9$ when $a_{1}=a_{2}=a_{3}=1 / 3$ and $\theta_{1}=\theta_{2}=\theta_{3}$. However, in this place we resolve this question from the point of view of the TI.

It should be emphasized that TI for a multipartite state actually defines a relative connection between the states that belong to different parties. For example, the GHZ state implies that all parties would have the same state simultaneously. This feature leads to the supposition that the nearest fully separable state would be also permutationally invariant. In contrast, the $W$ state implies that 
the nearest neighbor parties always have the same state [25], or equivalently that there is always a single party showing a state that is different from the other two parties. We should point out that this feature of the $W$ state is more fundamental than PI (see Sec. V), and it is a unique characteristic as compared to other TI multipartite states. It is thus a natural speculation that the nearest fully separable state should display the same TI. Thus we attain the important supposition for the nearest fully separable state for $|W\rangle$ :

$$
\begin{aligned}
\rho_{f}^{W}= & \frac{1}{3} \quad\left(\left|\phi\left(a_{1}, a_{2}=a_{3}, \theta_{1}, \theta_{2}=\theta_{3}\right)\right\rangle\left\langle\phi\left(a_{1}, a_{2}=a_{3}, \theta_{1}, \theta_{2}=\theta_{3}\right)\right|\right. \\
& +\left|\phi\left(a_{2}, a_{1}=a_{3}, \theta_{2}, \theta_{1}=\theta_{3}\right)\right\rangle\left\langle\phi\left(a_{2}, a_{1}=a_{3}, \theta_{2}, \theta_{1}=\theta_{3}\right)\right| \\
& \left.+\left|\phi\left(a_{3}, a_{1}=a_{2}, \theta_{3}, \theta_{1}=\theta_{2}\right)\right\rangle\left\langle\phi\left(a_{3}, a_{1}=a_{2}, \theta_{3}, \theta_{1}=\theta_{2}\right)\right|\right),
\end{aligned}
$$

where $|\phi\rangle$ and the independent variables $a_{n}, \theta_{n}(n=$ 1,2,3) are defined in Eq. (16), and three possible situations are set to be incoherent so that $\rho_{f}^{W}$ is separable. The crucial feature is in $\rho_{f}^{W}$ : that nearest-neighbor parties sharing the same state exist, which is same as that of $|W\rangle$. Furthermore, because all terms after cyclic translation are included, $\rho_{f}^{W}$ is obviously TI. Next we will display the correctness of this supposition by obtaining the same $\Lambda_{\max }$ by calculating the overlap of the $W$ state and $\rho_{f}^{W}$.

Similar to the determination of Eq. (15), the key point is to find the maximal overlaps of the $W$ state and the terms in $\rho_{f}^{W}$. Fortunately they are the same because of the same TI of the $W$ state and $\rho_{f}^{W}$, and then it is enough to evaluate the overlap with one arbitrary term in $\rho_{f}^{W}$, e.g.,

$$
\begin{aligned}
& \max \operatorname{Tr}\left[|W\rangle\langle W| \rho_{f}^{W}\right] \Leftrightarrow \\
& \frac{1}{3} \max \left|\left\langle W \mid \phi\left(a_{1}, a_{2}=a_{3}, \theta_{1}, \theta_{2}=\theta_{3}\right)\right\rangle\right|^{2} \\
\Rightarrow & \frac{1}{3} \max _{a_{1} \neq a_{2}, \theta_{1} \neq \theta_{2}} \mid \mathrm{e}^{2 i \theta_{2}} \sqrt{a_{1}\left(1-a_{2}\right)^{2}}+ \\
& \left.2 \mathrm{e}^{i\left(\theta_{1}+\theta_{2}\right)} \sqrt{\left(1-a_{1}\right) a_{2}\left(1-a_{2}\right)}\right|^{2} \\
\leq & \left.\frac{1}{3} \max _{a_{1} \neq a_{2}}\left[\sqrt{a_{1}\left(1-a_{2}\right)^{2}}+2 \sqrt{\left(1-a_{1}\right) a_{2}\left(1-a_{2}\right)}\right]^{2} 2\right)
\end{aligned}
$$

where the equality occurs when $\theta_{1}=\theta_{2}$.

The next step is to decide the maximal value of $\sqrt{a_{1}\left(1-a_{2}\right)^{2}}+2 \sqrt{\left(1-a_{1}\right) a_{2}\left(1-a_{2}\right)}$, which can be obtained easily by calculating its first and second derivation with independent variables $a_{1}, a_{2}$. Thus one can check that the maximal extremal point occurs at $a_{1}=a_{2}=$ $1 / 3$. Because $\rho_{3}^{W}$ is TI, the calculation for the other two components in Eq. (21) has the same result. Then the nearest FSPs are $\rho_{3}^{W}=\mid \phi\left(a_{1}=a_{2}=a_{3}=1 / 3, \theta_{1}=\right.$ $\left.\left.\theta_{2}=\theta_{3}\right)\right\rangle\left\langle\phi\left(a_{1}=a_{2}=a_{3}=1 / 3, \theta_{1}=\theta_{2}=\theta_{3}\right)\right|$, which demonstrates the validity of the supposition $\rho_{f}^{W}$.

Some comments are in order. The calculations of $\Lambda_{\max }$ for GHZ and $W$ states have illustrated the general procedure for determining $\Lambda_{\max }$ for TI entangled states. A crucial supposition is Eq.(21), which comes directly from our understanding of the TI in multipartite entangled states, and can be generalized easily to other multi-qubit states. Mathematically, in order to find the nearest fully separable state for the entangled state with a certain symmetry, it is enough to search into the state subspace with the same symmetry, because the overlap for the two states in the same subspace are believed to be no smaller than that in distinct spaces. Particularly, this supposition is not only valid for a pure state but is valid also for a mixed state, and a formal statement from the Schwartz-Cauchy inequality can be found in our recent work [9]. In the following discussion, we should demonstrate the popularity and validity of the supposition [Eq. (21)] through several exact examples.

\section{B. 4-qubit case}

There are four basic multipartite entangled states with TI: $|\mathrm{GHZ}\rangle_{4},|W\rangle_{4},\left|\mathrm{GHZ}^{\prime}\right\rangle_{4}$ and $|\psi\rangle_{4}=\frac{1}{2}(|1100\rangle+$ $|0110\rangle+|0011\rangle+|1001\rangle)[26]$. Because a general discussion on $|\mathrm{GHZ}\rangle_{N},|W\rangle_{N}$ and $\left|\mathrm{GHZ}^{\prime}\right\rangle_{N}$ will be presented at the end of this section, only $|\psi\rangle_{4}$ is studied here. The overlap is determined exactly by the relation

$$
\begin{aligned}
\left|{ }_{4}\langle\psi \mid \phi\rangle\right|^{2} & \frac{1}{4} \mid \mathrm{e}^{i\left(\theta_{3}+\theta_{4}\right)} \sqrt{a_{1} a_{2}\left(1-a_{3}\right)\left(1-a_{4}\right)}+\mathrm{e}^{i\left(\theta_{1}+\theta_{4}\right)} \times \\
& \sqrt{\left(1-a_{1}\right) a_{2} a_{3}\left(1-a_{4}\right)}+\mathrm{e}^{i\left(\theta_{1}+\theta_{2}\right)} \sqrt{\left(1-a_{1}\right)} \times \\
& \sqrt{\left(1-a_{2}\right) a_{3} a_{4}}+\left.\mathrm{e}^{i\left(\theta_{2}+\theta_{3}\right)} \sqrt{a_{1}\left(1-a_{2}\right)\left(1-a_{3}\right) a_{4}}\right|^{2} \\
= & \frac{1}{4} \mid\left(\mathrm{e}^{i \theta_{3}} \sqrt{a_{1}\left(1-a_{3}\right)}+\mathrm{e}^{i \theta_{1}} \sqrt{\left(1-a_{1}\right) a_{3}}\right) \times \\
\leq & \frac{1}{4}\left(\sqrt{a_{1}\left(1-a_{3}\right)}+\sqrt{\left(1-a_{1}\right) a_{3}}\right)^{2} \times \\
& \quad\left(\sqrt{a_{2}\left(1-a_{4}\right)}+\sqrt{\left(1-a_{2}\right) a_{4}}\right)^{2},
\end{aligned}
$$

where the last equality occurs when $\theta_{1}=\theta_{3}$ and $\theta_{2}=\theta_{4}$. It is easy to obtain $\Lambda_{\max }=1 / 4$ when $a_{1}+a_{3}=1$ and $a_{2}+a_{4}=1$. 
The TI of $|\psi\rangle_{4}$ is that there are two nearest-neighbor parties sharing one state, and the other two parties share a different state. With the above results, one could construct a nearest fully separable state,

$$
\begin{array}{r}
\rho_{f}^{|\psi\rangle_{4}}=\frac{1}{4}(|1100\rangle\langle 1100|+| 1001\rangle\langle 1001|+ \\
|0011\rangle\langle 0011|+| 0110\rangle\langle 0110|)
\end{array}
$$

with arbitrary $\theta_{n}(n=1,2,3,4)$ because they cancel each other out in this case. The above state obviously displays the same TI to $|\psi\rangle_{4}$. This result means that one can easily determine $\Lambda_{\max }$ by utilizing TI of $|\psi\rangle_{4}$.

An interesting case is the existence of the nearest FSPs with PI when $\theta_{1}=\theta_{2}=\theta_{3}=\theta_{4}$ and $a_{1}=a_{2}=a_{3}=$ $a_{4}=1 / 2$. In fact $|\psi\rangle_{4}$ is biseparable because $|\psi\rangle_{4}=$ $\frac{1}{\sqrt{2}}(|10\rangle+|01\rangle)_{13} \otimes \frac{1}{\sqrt{2}}(|10\rangle+|01\rangle)_{24}$. This feature implies that pairs 1-3 and 2-4 are uncorrelated completely, which forces $|\psi\rangle_{4}$ into a larger subspace than that of TI and PI. So the simultaneous existence of the fully separable state with PI and TI is not surprising in this case.

\section{5-qubit case}

Besides $|\mathrm{GHZ}\rangle_{5}$ and $|W\rangle_{5}$, there are two different basic TI entangled states

$$
\begin{aligned}
\left|\psi^{1 a}\right\rangle_{5} & =\frac{1}{\sqrt{5}}(|11000\rangle+|01100\rangle+|00110\rangle+|00011\rangle+\mid 10001 \\
\left|\psi^{1 b}\right\rangle_{5} & =\frac{1}{\sqrt{5}}(|10100\rangle+|01010\rangle+|00101\rangle+|10010\rangle+\mid 01001
\end{aligned}
$$

Unfortunately there are no exact results for $\Lambda_{\max }$ of $\left|\psi^{1 a(b)}\right\rangle_{5}$, so one has to rely on numerical evaluations.

The numerical procedure is to sample exhaustively the possibility of the values of independent variables as much as possible, and to record the maximal value of the overlap. Then when the numerical result is not changed for long sampling times, e.g., $10^{4}$ or so, this number is considered to be $\Lambda_{\max }$. For example, the overlap of $\left|\psi^{1 a}\right\rangle_{5}$ is

$$
\begin{aligned}
&\left|{ }_{5}\left\langle\psi^{1 a} \mid \phi\right\rangle\right|^{2} \\
&=\frac{1}{5} \mid \mathrm{e}^{i\left(\theta_{3}+\theta_{4}+\theta_{5}\right)} \sqrt{a_{1} a_{2}\left(1-a_{3}\right)\left(1-a_{4}\right)\left(1-a_{5}\right)}+ \\
& \mathrm{e}^{i\left(\theta_{1}+\theta_{4}+\theta_{5}\right)} \sqrt{\left(1-a_{1}\right) a_{2} a_{3}\left(1-a_{4}\right)\left(1-a_{5}\right)}+ \\
& \mathrm{e}^{i\left(\theta_{1}+\theta_{2}+\theta_{5}\right)} \sqrt{\left(1-a_{1}\right)\left(1-a_{2}\right) a_{3} a_{4}\left(1-a_{5}\right)}+ \\
& \mathrm{e}^{i\left(\theta_{1}+\theta_{2}+\theta_{3}\right)} \sqrt{\left(1-a_{1}\right)\left(1-a_{2}\right)\left(1-a_{3}\right) a_{4} a_{5}}+ \\
& \leq \frac{1}{5} {\left[\sqrt{a_{1} a_{2}\left(1-a_{3}\right)\left(1-a_{4}\right)\left(1-a_{5}\right)}+\sqrt{\left(1-a_{1}\right)} \times\right.} \\
& \sqrt{a_{2} a_{3}\left(1-a_{4}\right)\left(1-a_{5}\right)}+\sqrt{\left(1-a_{1}\right)\left(1-a_{2}\right)} \times \\
& \quad \sqrt{a_{3} a_{4}\left(1-a_{5}\right)}+\sqrt{\left(1-a_{1}\right)\left(1-a_{2}\right)\left(1-a_{3}\right) a_{4} a_{5}} \\
&\left.+\sqrt{a_{1}\left(1-a_{2}\right)\left(1-a_{3}\right)\left(1-a_{4}\right) a_{5}}\right]^{2}
\end{aligned}
$$

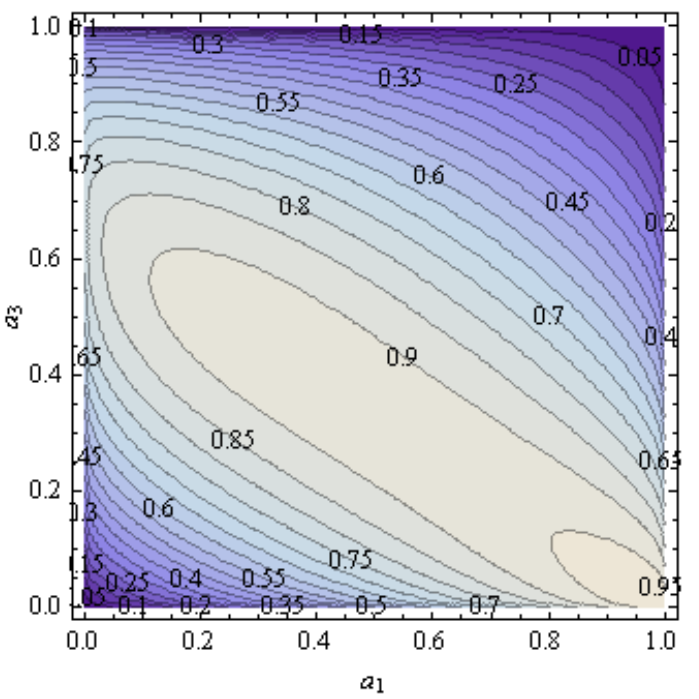

FIG. 2: (Color online) Contour plot of the function $f_{5}^{1}$ defined in Eq.(29).

where the second equality occurs when the values coincide for all $\theta_{\alpha}(\alpha=1,2,3,4,5)$. Thus the sampling can be reduced for $a_{\alpha}(\alpha=1,2,3,4,5)$. For reliability, the sampling times are chosen to be $10^{5}$ in this section. Finally the value is steadily closed to $1 / 5$ and $\left.\left.000 \operatorname{lid}_{|\phi\rangle}\right|_{5}\left\langle\psi^{1 a} \mid \phi\right\rangle\right|^{2} \rightarrow 1 / 5$.

01(2) Fityom the point of view of TI, the main feature of $\left|\psi^{1 a}\right\rangle_{5}$ is that two nearest-neighbor parties have the same state and the other parties share a different state. Thus an optimal supposition for the nearest FSPs is, similar to the method discussed in Sec. IIIA.

$$
a_{1}=a_{2}, a_{3}=a_{4}=a_{5} ; \theta_{1}=\theta_{2}, \theta_{3}=\theta_{4}=\theta_{5},
$$

It should be pointed that this choice is not unique because there are other different choices, similar to the ones adopted for Eq. (21). The validity of this supposition can be checked by asking whether one can obtain the same $\Lambda_{\max }$ by using the above numerical evaluation. For this purpose, one has

$$
\begin{gathered}
\quad\left|{ }_{5}\left\langle\psi^{1 a} \mid \phi\right\rangle\right|^{2} \\
=\frac{1}{5} \mid \mathrm{e}^{3 i \theta_{3}} a_{1}\left(1-a_{3}\right)^{3 / 2}+2 \mathrm{e}^{i\left(\theta_{1}+2 \theta_{3}\right)} \sqrt{a_{1}\left(1-a_{1}\right)} \times \\
\quad \sqrt{a_{3}\left(1-a_{3}\right)^{2}}+\left.2 \mathrm{e}^{i\left(2 \theta_{1}+\theta_{3}\right)} \sqrt{\left(1-a_{1}\right)^{2} a_{3}^{2}\left(1-a_{3}\right)}\right|^{2} \\
\leq \frac{1}{5}\left[a_{1}\left(1-a_{3}\right)^{3 / 2}+2 \sqrt{a_{1}\left(1-a_{1}\right) a_{3}\left(1-a_{3}\right)^{2}}\right. \\
\left.+2 \sqrt{\left(1-a_{1}\right)^{2} a_{3}^{2}\left(1-a_{3}\right)}\right]^{2}
\end{gathered}
$$


where the second equality occurs when $\theta_{1}=\theta_{3}$. Thus

$$
\begin{aligned}
\left.\left.\max _{|\phi\rangle}\right|_{5}\left\langle\psi^{1 a} \mid \phi\right\rangle\right|^{2} \Rightarrow & \\
\max _{a_{1}, a_{3}} f_{5}^{1}= & a_{1}\left(1-a_{3}\right)^{3 / 2}+2 \sqrt{a_{1}\left(1-a_{1}\right) a_{3}\left(1-a_{3}\right)^{2}} \\
& +2 \sqrt{\left(1-a_{1}\right)^{2} a_{3}^{2}\left(1-a_{3}\right)} .
\end{aligned}
$$

From Fig. 2, $f_{5}^{1}$ clearly has the maximal value 1 at $a_{1}=1$ and $a_{3}=0$, and then $\Lambda_{\max }$ is $1 / 5$ too.

This example displays the validity of the supposition Eq.(27). Then the nearest fully separable state with the same TI to $\left|\psi^{1 a}\right\rangle_{5}$ can be constructed readily, as was done in three- and four-qubit case. A similar discussion can also be applied to $\left|\psi^{1 b}\right\rangle_{5}$, for which $\Lambda_{\max }$ is also $1 / 5$. The difference is that the supposition for nearest FSPs from TI becomes $a_{1}=a_{3}, a_{2}=a_{4}=a_{5}$ and $\theta_{1}=\theta_{3}, \theta_{2}=\theta_{4}=$ $\theta_{5}$ in this case.

\section{6-qubit case}

In order to show further the fundamental role of the TI, the nearest FSPs and $\Lambda_{\max }$ for six-qubit multipartite entangled states are studied in this subsection. In addition to $|\mathrm{GHZ}\rangle_{6},|W\rangle_{6}$ and $\left|\mathrm{GHZ}^{\prime}\right\rangle_{6}$, there are three types of the basic TI entangled states:

$$
\begin{aligned}
\left|\psi^{1 a}\right\rangle_{6} & =\frac{1}{\sqrt{6}}(|110000\rangle+[\text { the other cyclic terms }]), \\
\left|\psi^{1 b}\right\rangle_{6} & =\frac{1}{\sqrt{6}}(|101000\rangle+[\text { the other cyclic terms }]), \\
\left|\psi^{2 a}\right\rangle_{6} & =\frac{1}{\sqrt{6}}(|111000\rangle+[\text { the other cyclic terms }]), \\
\left|\psi^{2 b}\right\rangle_{6} & =\frac{1}{\sqrt{6}}(|101100\rangle+[\text { the other cyclic terms }]), \\
\left|\psi^{2 c}\right\rangle_{6} & =\frac{1}{\sqrt{6}}(|110100\rangle+[\text { the other cyclic terms }]), \\
\left|\psi^{3}\right\rangle_{6} & =\frac{1}{\sqrt{3}}(|100100\rangle+|010010\rangle+|001001\rangle),
\end{aligned}
$$

which will be discussed respectively below.

1. $\left|\psi^{1 a}\right\rangle_{6}=\frac{1}{\sqrt{6}}(|110000\rangle+[$ the other cyclic terms $])$

There is no exact result of $\Lambda_{\max }$ for $\left|\psi^{1 a}\right\rangle$, and one has to rely on numerical evaluation, as was done in the previous subsection, which showed $\Lambda_{\max }=1 / 6$. Now let us repeat the calculation from the TI point of view. The crucial supposition for the nearest FSPs is $a_{1}=a_{2}, a_{3}=a_{4}=a_{5}=a_{6}$ and

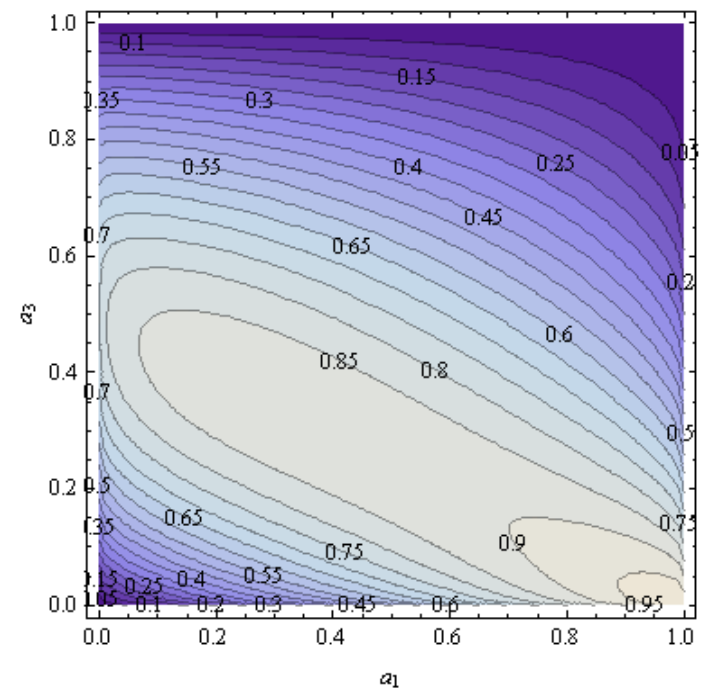

FIG. 3: (Color online) Contour plot of the function $f_{6}^{1}$ defined in Eq.(32).

$\theta_{1}=\theta_{2}, \theta_{3}=\theta_{4}=\theta_{5}=\theta_{6}$, and then

$$
\begin{aligned}
& \left.\quad{ }_{6}\left\langle\psi^{1 a} \mid \phi\right\rangle\right|^{2} \\
& =\frac{1}{6} \mid a_{1}\left(1-a_{3}\right)^{2} \mathrm{e}^{4 i \theta_{3}}+2 \sqrt{a_{1}\left(1-a_{1}\right) a_{3}\left(1-a_{3}\right)^{3}} \times \\
& \quad \mathrm{e}^{i\left(\theta_{1}+3 \theta_{3}\right)}+\left.3\left(1-a_{1}\right) a_{3}\left(1-a_{3}\right) \mathrm{e}^{2 i\left(\theta_{1}+\theta_{3}\right)}\right|^{2} \\
& \leq \frac{1}{6}\left[a_{1}\left(1-a_{3}\right)^{2}+2 \sqrt{a_{1}\left(1-a_{1}\right) a_{3}\left(1-a_{3}\right)^{3}}\right. \\
& \left.\quad+3\left(1-a_{1}\right) a_{3}\left(1-a_{3}\right)\right]^{2}
\end{aligned}
$$

where the second equality occurs when $\theta_{1}=\theta_{3}$. Then the determination of $\Lambda_{\max }$ is reduced to find the maximal values of

$$
\begin{aligned}
f_{6}^{1}= & a_{1}\left(1-a_{3}\right)^{2}+2 \sqrt{a_{1}\left(1-a_{1}\right) a_{3}\left(1-a_{3}\right)^{3}} \\
& +3\left(1-a_{1}\right) a_{3}\left(1-a_{3}\right) .
\end{aligned}
$$

As shown in Fig. 3, $f_{6}^{1}$ has a maximal value of 1 at $a_{1}=1, a_{3}=0$. Thus $\left|{ }_{6}\left\langle\psi^{1 a} \mid \phi\right\rangle\right|^{2}$ has a maximal value of $1 / 6$, which is the same as the numerical result. A similar study is also applied for $\left|\psi^{1 b}\right\rangle$ if we suppose $a_{1}=a_{3}, a_{2}=$ $a_{4}=a_{5}=a_{6}$, and $\theta_{1}=\theta_{3}, \theta_{2}=\theta_{4}=\theta_{5}=\theta_{6}$ in this case.

In conclusion, one can still find $\Lambda_{\max }$ efficiently by a TI of $\left|\psi^{1 a}\right\rangle_{6}$

2. $\left|\psi^{2 a}\right\rangle_{6}=\frac{1}{\sqrt{6}}(|111000\rangle+[$ the other cyclic terms $])$ 
After some simplifications, the overlap becomes

$$
\begin{aligned}
& \left|{ }_{6}\left\langle\psi^{2 a} \mid \phi\right\rangle\right|^{2} \leq \\
& \frac{1}{6}\left\{\sqrt { a _ { 3 } ( 1 - a _ { 6 } ) } \left[\sqrt{a_{1}\left(1-a_{4}\right) a_{2}\left(1-a_{5}\right)}+\sqrt{\left(1-a_{1}\right)} \times\right.\right. \\
& \left.\sqrt{a_{4}}\left(\sqrt{a_{2}\left(1-a_{5}\right)}+\sqrt{\left(1-a_{2}\right) a_{5}}\right)\right]+\sqrt{\left(1-a_{3}\right) a_{6}} \times \\
& {\left[\sqrt{\left(1-a_{1}\right) a_{4}\left(1-a_{2}\right) a_{5}}+\sqrt{a_{1}\left(1-a_{4}\right)}\left(\sqrt{a_{2}\left(1-a_{5}\right)}+\right.\right.} \\
& \left.\left.\left.\sqrt{\left(1-a_{2}\right) a_{5}}\right)\right]\right\}^{2}
\end{aligned}
$$

where the equality occurs when the value of $\theta_{\alpha}(\alpha=$ $1,2, \cdots, 6)$ coincides. By applying the inequalities

$$
\begin{aligned}
& \sqrt{a_{1}\left(1-a_{4}\right)} \leq \frac{a_{1}+\left(1-a_{4}\right)}{2}, \\
& \sqrt{a_{2}\left(1-a_{5}\right)} \leq \frac{a_{2}+\left(1-a_{5}\right)}{2}, \\
& \sqrt{a_{3}\left(1-a_{6}\right)} \leq \frac{a_{3}+\left(1-a_{6}\right)}{2},
\end{aligned}
$$

where the equality occurs iff $a_{1}+a_{4}=1, a_{2}+a_{5}=1$ and $a_{3}+a_{6}=1$. Then

$$
\left|{ }_{6}\left\langle\psi^{2 a} \mid \phi\right\rangle\right|^{2} \Rightarrow \leq \frac{1}{6}\left(1-a_{2}+a_{1} a_{2}+a_{2} a_{3}-a_{1} a_{3}\right)^{2} .
$$

It is easy for the above expression to find the unique extremal point at $a_{1}=a_{2}=a_{3}=1 / 2$, and then the extremal value for $\left.\left.\right|_{6}\left\langle\psi^{2 a} \mid \phi\right\rangle\right|^{2}$ is $\frac{3}{32}$. However it is known that the extremal value is not completely equivalent to the maximal, and so the boundary points when $a_{i}=1,0(i=1,2, \cdots, N)$ have to be checked independently. One can find the overlap $1 / 6$ at the boundary point $a_{1}=a_{2}=a_{3}=1, a_{4}=a_{5}=a_{6}=0$, for example. Because the extremal point is unique, $\Lambda_{\max }$ in this case is just $1 / 6$. This exact result is clearly consistent with the TI structure of $\left|\psi^{2 a}\right\rangle_{6}$; there are always three nearest-neighbor parties having the same state and the other parties sharing one different state.

$$
\text { 3. }\left|\psi^{3}\right\rangle_{6}=\frac{1}{\sqrt{3}}(|100100\rangle+|010010\rangle+|001001\rangle)
$$

The overlap is

$$
\begin{aligned}
&\left|{ }_{6}\left\langle\psi^{3} \mid \phi\right\rangle\right|^{2} \\
&= \frac{1}{3} \mid \mathrm{e}^{i\left(\theta_{2}+\theta_{3}+\theta_{5}+\theta_{6}\right)} \sqrt{a_{1}\left(1-a_{2}\right)\left(1-a_{3}\right) a_{4}\left(1-a_{5}\right)} \times \\
& \sqrt{1-a_{6}}+\mathrm{e}^{i\left(\theta_{1}+\theta_{3}+\theta_{4}+\theta_{6}\right)} \sqrt{\left(1-a_{1}\right) a_{2}\left(1-a_{3}\right)} \times \\
& \quad \sqrt{\left(1-a_{4}\right) a_{5}\left(1-a_{6}\right)}+\mathrm{e}^{i\left(\theta_{1}+\theta_{2}+\theta_{4}+\theta_{5}\right)} \sqrt{\left(1-a_{1}\right)} \times \\
& \leq\left.\frac{1}{\left(1-a_{2}\right) a_{3}\left(1-a_{4}\right)\left(1-a_{5}\right) a_{6}}\right|^{2} \\
& \leq \sqrt{a_{1}\left(1-a_{2}\right)\left(1-a_{3}\right) a_{4}\left(1-a_{5}\right)\left(1-a_{6}\right)}+ \\
&=\frac{1}{3}\left\{\sqrt{\left(1-a_{1}\right) a_{2}\left(1-a_{3}\right)\left(1-a_{4}\right) a_{5}\left(1-a_{6}\right)}+\right. \\
&\left.\quad+\sqrt{\left(1-a_{1}\right)\left(1-a_{2}\right) a_{3}\left(1-a_{4}\right)\left(1-a_{5}\right) a_{6}}\right]^{2} \\
& \quad \sqrt{\left(1-a_{6}\right)}\left[\sqrt{a_{1} a_{4}\left(1-a_{2}\right)\left(1-a_{5}\right)}\right.
\end{aligned}
$$

where the second equality occurs when $\theta_{1}+\theta_{4}=\theta_{2}+\theta_{5}=$ $\theta_{3}+\theta_{6}$. From the relations $a b \leq\left(a^{2}+b^{2}\right) / 2$, the above equation becomes

$$
\begin{aligned}
\Rightarrow \leq & \left\{\left[a_{1}\left(1-a_{2}\right)+\left(1-a_{1}\right) a_{2}\right]\left(1-a_{3}\right)+\right. \\
& \left.\left(1-a_{1}\right)\left(1-a_{2}\right) a_{3}\right\}^{2},
\end{aligned}
$$

of which the extremal point appears at $a_{1}=a_{2}=a_{3}=$ $a_{0}$. In this case the unique maximal extremal value of $\left|{ }_{6}\left\langle\psi^{3} \mid \phi\right\rangle\right|^{2}$ is $16 / 3^{5}$ for $a_{0}=1 / 3$, while at the boundary $a_{1}=1, a_{2}=a_{3}=0$ the overlap is $1 / 3>16 / 3^{5}$. Finally one obtains $\Lambda_{\max }=1 / 3$ when $a_{1}=a_{4}=1, a_{2}=a_{3}=$ $a_{5}=a_{6}=0$, for example. With respect to the flexible limit on $\theta_{\alpha}$, the FPSs can be constructed readily with the same TI to $\left|\psi^{3}\right\rangle_{6}$.

\section{E. 8-qubit case}

As for seven-qubit case, the discussion is similar to that of the five-qubit cases. Because there are no exact results for $\lambda_{\max }$ of seven-qubit entangled states, except for $|\mathrm{GHZ}\rangle_{7}$ and $|W\rangle_{7}$, this situation is ignored in this section and instead we focus on the eight-qubit case.

The situation becomes complex for eight-qubit multipartite entangled states because there are several special cyclic structures in this case. We will focus on these interesting situations because e the exact results can be obtained.

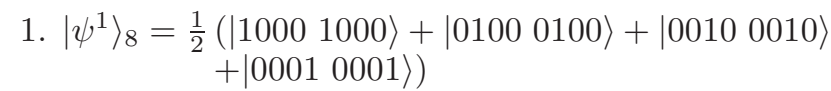


The overlap is

$$
\begin{aligned}
& \left|{ }_{8}\left\langle\psi^{1} \mid \psi\right\rangle\right|^{2} \\
= & \frac{1}{4} \mid \mathrm{e}^{i \sum_{\alpha \neq 1,5}^{8} \theta_{\alpha}} \sqrt{a_{1} a_{5}} \prod_{\alpha \neq 1,5}^{8} \sqrt{1-a_{\alpha}}+\mathrm{e}^{i \sum_{\alpha \neq 2,6}^{8} \theta_{\alpha}} \times \\
& \sqrt{a_{2} a_{6}} \prod_{\alpha \neq 2,6}^{8} \sqrt{1-a_{\alpha}}+\mathrm{e}^{i \sum_{\alpha \neq 3,7}^{8} \theta_{\alpha}} \sqrt{a_{3} a_{7}} \times \\
\leq & \frac{1}{4}\left[\sqrt{a_{1} a_{5}} \prod_{\alpha \neq 1,5}^{8} \sqrt{1-a_{\alpha}}+\sqrt{a_{2} a_{6}} \prod_{\alpha \neq 2,6}^{8} \sqrt{1-a_{\alpha}}\right. \\
& \left.+\sqrt{a_{3} a_{7}} \prod_{\alpha \neq 3,7}^{8} \sqrt{1-a_{\alpha}}+\sqrt{a_{4} a_{8}} \prod_{\alpha \neq 4,8}^{8} \sqrt{1-a_{\alpha}}\right]^{2}(3,8)
\end{aligned}
$$

where the second equality occurs when $\theta_{1}+\theta_{5}=\theta_{2}+\theta_{6}=$ $\theta_{3}+\theta_{7}=\theta_{4}+\theta_{8}$. The above equation can be rewritten as

$$
\begin{aligned}
\Rightarrow \frac{1}{4}\{ & \left\{\sqrt{a_{1} a_{5}\left(1-a_{2}\right)\left(1-a_{6}\right)}+\sqrt{\left(1-a_{1}\right)\left(1-a_{5}\right)} \times\right. \\
& \left.\sqrt{a_{2} a_{6}}\right] \sqrt{\left(1-a_{3}\right)\left(1-a_{4}\right)\left(1-a_{7}\right)\left(1-a_{8}\right)}+ \\
& {\left[\sqrt{a_{3} a_{7}\left(1-a_{4}\right)\left(1-a_{8}\right)}+\sqrt{\left(1-a_{3}\right)\left(1-a_{7}\right)} \times\right.} \\
& \left.\left.\sqrt{a_{4} a_{8}}\right] \sqrt{\left(1-a_{1}\right)\left(1-a_{5}\right)\left(1-a_{2}\right)\left(1-a_{6}\right)}\right\}^{2} \\
\leq \frac{1}{4}\{ & \left\{a_{1}\left(1-a_{2}\right)+\left(1-a_{1}\right) a_{2}\right]\left(1-a_{3}\right)\left(1-a_{4}\right)+ \\
& \left.\left(1-a_{1}\right)\left(1-a_{2}\right)\left[a_{3}\left(1-a_{4}\right)+\left(1-a_{3}\right) a_{4}\right]\right\}^{2}
\end{aligned}
$$

where the second equality occurs when $a_{1}=a_{5}, a_{2}=$ $a_{6}, a_{3}=a_{7}$ and $a_{4}=a_{8}$ by the inequality $a^{2}+b^{2} \geq 2|a b|$.

One can check easily that the extremal point of the above equation appears when $a_{1}=a_{2}=a_{3}=a_{4}=a_{0}$, and then the maximal extremal value is $3^{6} / 4^{7}$ when $a_{0}=1 / 4$. As for the boundary, one can directly find the overlap $1 / 4>3^{6} / 4^{7}$ only if $a_{\alpha}=1$ for any one of $\alpha=1,2,3,4$, and the other is zero. Then $\Lambda_{\max }=1 / 4$. In addition this situation is consistent with the TI of $\left|\psi^{1}\right\rangle_{8}$; two single-party states, four-party separated from each other, always have the same state and the others share another different state.

$$
\text { 2. } \begin{aligned}
\left|\psi^{2}\right\rangle_{8}= & \frac{1}{2}(|11001100\rangle+|01100110\rangle+|00110011\rangle \\
& +|10011001\rangle)
\end{aligned}
$$

The overlap is

$$
\begin{aligned}
&\left|{ }_{8}\left\langle\psi^{2} \mid \psi\right\rangle\right|^{2} \\
&= \frac{1}{4} \mid \mathrm{e}^{i\left(\theta_{3}+\theta_{4}+\theta_{7}+\theta_{8}\right)} \sqrt{a_{1} a_{2}\left(1-a_{3}\right)\left(1-a_{4}\right) a_{5} a_{6}} \times \\
& \sqrt{\left(1-a_{7}\right)\left(1-a_{8}\right)}+\mathrm{e}^{i\left(\theta_{1}+\theta_{4}+\theta_{5}+\theta_{8}\right)} \sqrt{\left(1-a_{1}\right)} \times \\
& \sqrt{a_{2} a_{3}\left(1-a_{4}\right)\left(1-a_{5}\right) a_{6} a_{7}\left(1-a_{8}\right)}+ \\
& \mathrm{e}^{i\left(\theta_{1}+\theta_{2}+\theta_{5}+\theta_{6}\right)} \sqrt{\left(1-a_{1}\right)\left(1-a_{2}\right) a_{3} a_{4}\left(1-a_{5}\right)} \times \\
& \quad \sqrt{\left(1-a_{6}\right) a_{7} a_{8}}+\mathrm{e}^{i\left(\theta_{2}+\theta_{3}+\theta_{6}+\theta_{7}\right)} \sqrt{a_{1}\left(1-a_{2}\right)} \times \\
& \leq \frac{1}{4}\left[\sqrt{a_{1} a_{2}\left(1-a_{3}\right)\left(1-a_{4}\right) a_{5} a_{6}\left(1-a_{7}\right)\left(1-a_{8}\right)}+\right. \\
& \quad \sqrt{\left(1-a_{1}\right) a_{2} a_{3}\left(1-a_{4}\right)\left(1-a_{5}\right) a_{6} a_{7}\left(1-a_{8}\right)}+ \\
& \quad \sqrt{\left(1-a_{1}\right)\left(1-a_{2}\right) a_{3} a_{4}\left(1-a_{5}\right)\left(1-a_{6}\right) a_{7} a_{8}}+ \\
&\left.\quad \sqrt{a_{1}\left(1-a_{2}\right)\left(1-a_{3}\right) a_{4} a_{5}\left(1-a_{6}\right)\left(1-a_{7}\right) a_{8}}\right]^{2}(40)
\end{aligned}
$$

where the second equality occurs when $\theta_{1}+\theta_{5}=\theta_{2}+\theta_{6}=$ $\theta_{3}+\theta_{7}=\theta_{4}+\theta_{8}$.

The above equation can be rewritten as

$$
\begin{aligned}
\Rightarrow & \frac{1}{4}\left[\sqrt{a_{1}\left(1-a_{3}\right) a_{5}\left(1-a_{7}\right)}+\sqrt{\left(1-a_{1}\right) a_{3}\left(1-a_{5}\right) a_{7}}\right]^{2} \\
& \times\left[\sqrt{a_{2}\left(1-a_{4}\right) a_{6}\left(1-a_{8}\right)}+\sqrt{\left(1-a_{2}\right) a_{4}\left(1-a_{6}\right) a_{8}}\right]^{2} \\
\leq & \frac{1}{4}\left[a_{1}\left(1-a_{3}\right)+\left(1-a_{1}\right) a_{3}\right]^{2}\left[a_{2}\left(1-a_{4}\right)+\left(1-a_{2}\right) a_{(4}{ }_{1}^{2}\right)
\end{aligned}
$$

where the second equality occurs when $a_{1}=a_{5}, a_{2}=$ $a_{6}, a_{3}=a_{7}$ and $a_{4}=a_{8}$. The extremal point of the equation above is uniquely at $a_{1}=a_{2}=a_{3}=a_{4}=1 / 2$, at which the maximal extremal value is $1 / 2^{6}$. However one can easily find that the overlap is $1 / 4$ when $a_{1}=$ $1, a_{3}=0$ and $a_{2}=1, a_{4}=0$, for example, which is obviously larger than $1 / 2^{6}$. Thus $\Lambda_{\max }=1 / 4$ in this case.

The condition $a_{1}=a_{2}=1$ and $a_{3}=a_{4}=0$ coincides with the TI structure of $\left|\psi^{2}\right\rangle_{8}$; there are two pairs of nearest- neighbor parties, two-party separated from each other, always having the same state. This observation again displays the underlying effect of the TI structure of the entangled state on the determination of the maximal overlap $\Lambda_{\max }$.

Actually $\left|\psi^{2}\right\rangle_{8}$ is biseparable because $\left|\psi^{2}\right\rangle_{8}=$ $\frac{1}{\sqrt{2}}(|1010\rangle+|0101\rangle)_{1357} \otimes \frac{1}{\sqrt{2}}(|1010\rangle+|0101\rangle)_{2468}$. The above result is also the manifestation of this biseparable structure.

\section{F. Arbitrary $N$-qubit case: exact results}

For arbitrary $N$-qubit multipartite states, the maximal overlap can be determined exactly only for some special cases. However, the TI structure would still play a fundamental role in the evaluation of $\Lambda_{\max }$, as shown 
in the following discussions.

1. $\left|\psi_{\mathrm{GHZ}}\right\rangle_{N}=\sqrt{c}|11 \cdots 1\rangle+\mathrm{e}^{i \varphi} \sqrt{1-c}|00 \cdots 0\rangle$,

where $c \in[0,1]$ and $\varphi \in[0,2 \pi)$. It becomes $|\mathrm{GHZ}\rangle_{N}$ when $c=1 / 2$ and $\varphi=0$. The overlap is

$$
\begin{aligned}
& \left|{ }_{N}\left\langle\psi_{\mathrm{GHZ}} \mid \phi\right\rangle\right|^{2} \\
= & \left|\sqrt{c} \prod_{n=1}^{N} \sqrt{a_{n}}+\mathrm{e}^{i\left(-\varphi+\sum_{n=1}^{N} \theta_{n}\right)} \sqrt{1-c} \prod_{n=1}^{N} \sqrt{1-a_{n}}\right|^{2} \\
\leq & {\left[\sqrt{c} \prod_{n=1}^{N} \sqrt{a_{n}}+\sqrt{1-c} \prod_{n=1}^{N} \sqrt{1-a_{n}}\right]^{2} } \\
\leq & {\left[\sqrt{c} a_{0}^{N / 2}+\sqrt{1-c}\left(1-a_{0}\right)^{N / 2}\right]^{2} }
\end{aligned}
$$

where the second equality occurs when $\sum_{n=1}^{N} \theta_{n}=\varphi$, and the third one occurs when $a_{n}=a_{0}$ for arbitrary $n=1,2, \cdots, N$.

The extremal point of the above equation is determined by the relation

$$
\left(\frac{1}{a_{0}}-1\right)^{\frac{N}{2}-1}=\sqrt{\frac{c}{1-c}}
$$

which however corresponds to the minimal extremal value. Thus the maximal overlap can appear only at the boundary points $a_{0}=1$ or $a_{0}=0$ and $\Lambda_{\max }=c$ or $(1-c)$, depending on $c>1-c$ or $c<1-c$. Only if one choose $\theta_{n}=\varphi / N$ for arbitrary $n=1,2, \cdots, N$, the resultant fully separable state is also PI.

2. $\left|\psi_{\mathrm{GHZ}}^{\prime}\right\rangle_{N}=\sqrt{c}|1010 \cdots 10\rangle+\mathrm{e}^{i \varphi} \sqrt{1-c}|0101 \cdots 01\rangle$ $($ even $N)$

Obviously $\left|\psi_{\mathrm{GHZ}}^{\prime}\right\rangle_{N}$ and $\left|\psi_{\mathrm{GHZ}}\right\rangle_{N}$ can be converted into each other by the local unitary operation $\sigma_{2}^{x} \otimes \sigma_{4}^{x} \otimes \cdots \otimes$ $\sigma_{2 n}^{x} \otimes \cdots$, and the two states have the same measure of entanglement. However, the former displays a different TI from the latter because the operation is only imposed on the even sites [26]. Generally if two states can be related by local unitary transformation, it implies only that they have the same measure of entanglement, but not that the nearest fully separable state can be determined trivially in the same way.

This state becomes $\left|\mathrm{GHZ}^{\prime}\right\rangle_{N}$ when $c=1 / 2$ and $\varphi=0$. Its overlap is

$$
\begin{aligned}
& \left|{ }_{N}\left\langle\psi_{\mathrm{GHZ}}^{\prime} \mid \phi\right\rangle\right|^{2} \\
= & \mid \mathrm{e}^{i \sum_{n=1}^{N / 2} \theta_{2 n}} \sqrt{c} \prod_{n=1}^{N / 2} \sqrt{a_{2 n-1}} \prod_{n=1}^{N / 2} \sqrt{1-a_{2 n}}+ \\
& \left.\mathrm{e}^{i\left(-\varphi+\sum_{n=1}^{N / 2} \theta_{2 n-1}\right)} \sqrt{1-c} \prod_{n=1}^{N / 2} \sqrt{1-a_{2 n-1}} \prod_{n=1}^{N / 2} \sqrt{a_{2 n}}\right|^{2} \\
\leq & {\left[\sqrt{c} \prod_{n=1}^{N / 2} \sqrt{a_{2 n-1}} \prod_{n=1}^{N / 2} \sqrt{1-a_{2 n}}+\sqrt{1-c} \times\right.} \\
& \left.\prod_{n=1}^{N / 2} \sqrt{1-a_{2 n-1}} \prod_{n=1}^{N / 2} \sqrt{a_{2 n}}\right]^{2} \\
\leq & {\left[\sqrt{c} a_{1}^{\frac{N}{4}}\left(1-a_{2}\right)^{\frac{N}{4}}+\sqrt{1-c}\left(1-a_{1}\right)^{\frac{N}{4}} a_{2}^{\frac{N}{4}}\right]^{2} }
\end{aligned}
$$

where the second equality occurs when $\sum_{n=1}^{N / 2}\left(\theta_{2 n-1}-\theta_{2 n}\right)=\varphi$, and the third one occurs when $a_{2 n-1}=a_{1}$ and $a_{2 n}=a_{2}$ for $n=1,2, \cdots, N / 2$ by Eq.(18).

The above equation can be simplified further as

$$
\Rightarrow \leq\left[\sqrt{c} a_{1}^{\frac{N}{2}}+\sqrt{1-c}\left(1-a_{1}\right)^{\frac{N}{2}}\right]^{2},
$$

where the equality occurs when $a_{1}=1-a_{2}$. There is a minimal extremal point for the above equation, decided by

$$
\left(\frac{1}{a_{1}}-1\right)^{\frac{N}{2}-1}=\sqrt{\frac{c}{1-c}}
$$

Thus $\Lambda_{\max }=c$ or $(1-c)$, depending on $c>1-c$ or $c<1-c$, appears when $a_{1}=1, a_{2}=0$ or $a_{1}=0, a_{2}=1$. Finally, because the choice for $\theta_{2 n}$ and $\theta_{2 n-1}$ depends flexibly on the relation $\sum_{n=1}^{N / 2}\left(\theta_{2 n-1}-\theta_{2 n}\right)=\varphi$, one could freely choose $\theta_{2 n-1}=\theta_{1}, \theta_{2 n}=\theta_{2}$, and $\theta_{1} \neq \theta_{2}$. Thus one has the nearest FSPs, which show the same TI structure to $\left|\psi_{\mathrm{GHZ}}^{\prime}\right\rangle_{N}$.

Some comments should be made about $\left|\mathrm{GHZ}^{\prime}\right\rangle_{N}$ because there are some ambiguities and disputs about its nearest FSPs [17]. The crucial difference between $\left|\mathrm{GHZ}^{\prime}\right\rangle_{N}$ and $\left|\psi_{\mathrm{GHZ}}^{\prime}\right\rangle_{N}$ is at the TI; $\left|\mathrm{GHZ}^{\prime}\right\rangle_{N}$ is TI exactly because $|1010 \cdots 10\rangle$ and $|0101 \cdots 01\rangle$ occur with the same probability amplitude, while the basic feature of $\left|\psi_{\mathrm{GHZ}}^{\prime}\right\rangle_{N}$ is that the states are the same for the nextnearest-neighbor parties. Because $\left|\mathrm{GHZ}^{\prime}\right\rangle_{N}$ is a special case of $\left|\psi_{\mathrm{GHZ}}^{\prime}\right\rangle_{N}$, both states then share this basic feature. Following the discussion above, the common nearest FSPs are $|1010 \cdots 10\rangle$ or $|0101 \cdots 01\rangle$. However, we emphasize that this result proves exactly the correctness of the supposition for the nearest fully separable state, based on the TI structure of $\left|\mathrm{GHZ}^{\prime}\right\rangle_{N}$ as discussed in Sec. III 


$$
\begin{aligned}
\rho_{\mathrm{GHZ}^{\prime}}^{F S P s}= & \frac{1}{2}\left(\left|\phi\left(a_{2 n+1}=a, a_{2 n}=b, \theta_{2 n+1}=\alpha, \theta_{2 n}=\beta\right)\right\rangle\left\langle\phi\left(a_{2 n+1}=a, a_{2 n}=b, \theta_{2 n+1}=\alpha, \theta_{2 n}=\beta\right)\right|\right. \\
& \left.+\left|\phi\left(a_{2 n+1}=b, a_{2 n}=a, \theta_{2 n+1}=\beta, \theta_{2 n}=\alpha\right)\right\rangle\left\langle\phi\left(a_{2 n+1}=b, a_{2 n}=a, \theta_{2 n+1}=\beta, \theta_{2 n}=\alpha\right)\right|\right)
\end{aligned}
$$

Then the nearest fully separable state, from the above exact result,

$$
\frac{1}{2}(|1010 \cdots 10\rangle\langle 1010 \cdots 10|+| 0101 \cdots 01\rangle\langle 0101 \cdots 01|),
$$

displays the same TI to $\left|\mathrm{GHZ}^{\prime}\right\rangle_{N}$.

3. $|W\rangle_{N}=\frac{1}{\sqrt{N}}(|10 \cdots 0\rangle+|010 \cdots 0\rangle+\cdots+|0 \cdots 01\rangle)$

The overlap is

$$
\begin{aligned}
& \left|{ }_{N}\langle W \mid \phi\rangle\right|^{2} \\
= & \frac{1}{N}\left|\mathrm{e}^{i \sum_{n=1}^{N} \theta_{n}}\left(\prod_{n=1}^{N} \sqrt{1-a_{n}}\right) \sum_{n=1}^{N} \sqrt{\frac{a_{n}}{1-a_{n}}} \mathrm{e}^{-i \theta_{n}}\right|^{2} \\
\leq & \frac{1}{N}\left[\left(\prod_{n=1}^{N} \sqrt{1-a_{n}}\right) \sum_{n=1}^{N} \sqrt{\frac{a_{n}}{1-a_{n}}}\right]^{2},
\end{aligned}
$$

where the second equality occurs when the values of all $\theta_{n}$ coincide. Then it is enough to find the maximal values of

$$
f_{W}=\left(\prod_{n=1}^{N} \sqrt{1-a_{n}}\right) \sum_{n=1}^{N} \sqrt{\frac{a_{n}}{1-a_{n}}} .
$$

First let us find the extremal point for $f_{W}$, determined by

$$
\frac{\partial f_{W}}{\partial a_{k}}=0 \Rightarrow \sum_{n=1}^{N} \sqrt{\frac{a_{n}}{1-a_{n}}}=\frac{1}{\sqrt{a_{k}\left(1-a_{k}\right)}}
$$

Because the relation above is satisfied for arbitrary $a_{n}$, the extremal point occurs only if $a_{n}=a_{0}$ for arbitrary $n=1,2, \cdots, N$. Instead, to find the second derivative with $a_{n}$, it is convenient to evaluate the maximal value of

$$
f_{W}^{\prime}=N a_{0}\left(1-a_{0}\right)^{N-1}
$$

which is the overlap for $a_{n}=a_{0}$ with $n=1,2, \cdots, N$. It is easy to find the extremal value of $f_{W}^{\prime}$ when $a_{0}=1 / N$. From the second derivation,

$$
\frac{\partial^{2} f_{W}^{\prime}}{\partial a_{0}^{2}}=(N-1)\left(1-a_{0}\right)^{N-1}\left(N a_{0}-2\right),
$$

thus $a_{0}=1 / N$ is a maximal extremal point and

$$
\max f_{W}^{\prime}=\left(1-\frac{1}{N}\right)^{N-1} .
$$

As for the boundary where the overlap is $1 / N$, one can check that $\left(1-\frac{1}{N}\right)^{N-1}-\frac{1}{N} \geq 0$ for $N \geq 2$.

In conclusion, $\Lambda_{\max }=\left(1-\frac{1}{N}\right)^{N-1}$ when $a_{n}=a_{0}=$ $1 / N$ and $\theta_{n}=\theta_{0}$ for arbitrary $n=1,2, \cdots, N$. Thus the nearest fully separable state is also PI.

\section{PERIODIC STRUCTURE OF TRANSLATIONALLY INVARIANT ENTANGLED STATE}

It is obvious from previous studies that the optimal determination of $\Lambda_{\max }$ can be reduced greatly by utilizing the TI of multipartite entangled states. This result is based on the observation that the TI of a multipartite state actually defines the connection between the singleparty states in an unique manner, and, moreover, this connection can be spread over all single-party states because of TI. It should be pointed out that TI is a distinct feature from one to the other state; for example, GHZ and $W$ states show different TIs by which one can easily distinguish one from the other. Thus it is conjectured that a nearest fully separable state would also exist that can manifest a unique TI of the multipartite state. As shown in this section, this state can be constructed easily by combining all nearest FSPs incoherently with the same amplitude. Thus we claim that there exists a nearest fully separable state with the same TI as that of an entangled state, though it may be mixed generally.

Focusing on the geometry of the circle as shown in Fig. [1. there are diverse cyclic features in the basic TI entangled states studied in the previous section. For example, the distinct character for $\left|\psi_{\mathrm{GHZ}}\right\rangle_{N}$ is that all parties share the same state. From this point of view, depicted in Fig. 1. it is enough to translate the whole system only once in order that the state reverted to its original form. Thus we say it is one period, whereas for $\left|\mathrm{GHZ}^{\prime}\right\rangle_{N}$ the single-party states for next-nearest-neighbored parties are always the same, and $\left|\mathrm{GHZ}^{\prime}\right\rangle_{N}$ is two period because the state has to translate twice back to its original form, which could be envisioned also from Fig. 1] This is also the main reason that $\left|\mathrm{GHZ}^{\prime}\right\rangle_{N}$ is composed of two terms. Consequently $|W\rangle_{N}$ is $N$ period because one has to translate the system $N$ times in order to span all possible forms. In addition $\left|\psi^{3}\right\rangle_{6}$ is three period, and both of $\left|\psi^{1(2)}\right\rangle_{8}$ are four period.

An important finding from the above discussion is that the period numbers seem to be the common divisors of the party number $N$. Besides the trivial common divisors 1 and $N$, for example there are two- and three-period TI entangled states for the six-qubit system because $6=$ $2 \times 3$. For eight-qubit case, there are two- and four-period TI entangled state because $8=2 \times 4$. Because the spatial of the state is a circle, it is not strange that TI is heavily dependent on the number of party.

In conclusion, for a $(N=n \times m)$-qubit system, there always exist $n$ - and $m$-period TI entangled states. Thus by exploring the common divisors for $N$, one can find 
all possible cyclic structures of the $N$-qubit TI entangled state. As for prime $N$, there are only trivial oneand $N$-period states, as shown for the five-qubit system. Furthermore, we point out that one can construct more complicated TI entangled states principally by combining the basic TI entangled states with different periodic structures; for example, the Dicke state for the four-qubit system $|S(4 ; 2)\rangle$, defined in Eq. (7), can be decomposed into $|S(4 ; 2)\rangle=\frac{1}{\sqrt{3}}\left|\mathrm{GHZ}^{\prime}\right\rangle_{4}+\sqrt{\frac{2}{3}}|\psi\rangle_{4}$. It is the reason that we call them basic TI entangled states, which we studied in previous section.

An entangled state composed of different TI structures is called a hybrid. Then an important question is how to find the nearest fully separable state for the hybrid TI entangled states. And it is more interesting whether $\Lambda_{\max }$ is related to the different TIs in this state. If the answer is yes, it would simplify greatly the determination of $\Lambda_{\max }$. In the next section, we would present an explicit study of this question.

\section{FINDING THE MAXIMAL OVERLAP II: HYBRID TRANSLATIONAL INVARIANCE AND THE HIERARCHY OF THE PERIODIC STRUCTURE}

For hybrid TI entangled states, $\Lambda_{\max }$ is in general difficult to evaluate analytically. By presenting several numerical examples in this section, we try to illustrate some general features for these types of states, which are helpful in reducing the optimal determination for $\Lambda_{\max }$ in this case.

Unfortunately, we cannot provide an exact result to solidify the conclusions summarized at the end of this section, and instead have to rely on the numerical evaluation. For simplicity, the examples below will focus on the hybrid TI entangled states (HTIEs), composed of only two basic entangled states with different TIs. The goal is to find the effect of TI in HTIEs on the optimal determinations of $\Lambda_{\max }$ and the nearest FSPs. In addition, a hierarchy for the basic TI entangled states can be found.

The numerical procedure is stated below. Because the HTIEs in this section includes only two different TI structures, it is natural to suppose that the nearest FSPs would be determined by one of the two periodic structures. This supposition comes directly from the discussion in Sec III. However the supposed nearest fully separable states have to include two TIs. Then two numerical evaluations, called case 1 and case 2 , are implemented respectively for two optimally supposed nearest FSPs based on the TIs in HTIEs. The other two situations, called case 0 and case 3, correspond respectively to no supposition and the PI supposition for the nearest FSPs in order to check the validity of the two former suppositions.

The numerical procedure is the same as discussed in Sec. III C, we sample randomly the permissible values of all variables as much as possible and find the maximal overlap, which is unchanged for $10^{4}$ sampling times or so. The critical problem is to justify which supposition is optimal. Our method is to find one of the suppositions, for which the numerical result of the overlap is maximal after the same sampling times. Then we claim that this case is just the optimal requirement for the nearest FSPs in order to determine $\Lambda_{\max }$. The reason is simple; it is known that the random sampling evaluation could attain the same value theoretically only if the sampling times are infinite. With finite sampling times, the optimal one is believed to attain the maximal value more quickly than the nonoptimal sampling time. The sampling times are $10^{5}$ in order to guarantee the reliability of the numerical result and to control the time of evaluation. We should emphasize that this numerical evaluation is to show the effect of TI of multipartite entangled states on the determinations of FSPs and $\Lambda_{\max }$ rather than finding the exact results, because the sampling times are far from exhaustive. More details of the calculation are shown with special examples.

\section{A. 3-qubit case}

For the three-qubit system, there are only two basic TI entangled states: $|\mathrm{GHZ}\rangle$ of one-period and $|W\rangle$ of threeperiod. It is known that the nearest fully separable states for the two states are both permutationally invariant. Thus for their HTIEs, the nearest FSPs are definitely permutationally invariant also. So this case is omitted in this section.

\section{B. 4-qubit case}

There are one, two and four-period basic TI entangled states in this case, $|\mathrm{GHZ}\rangle_{4},\left|\mathrm{GHZ}^{\prime}\right\rangle_{4}$ and $|W\rangle_{4},|\psi\rangle_{4}$. Then there are five different HTIEs:

$$
\begin{aligned}
& \mathrm{A} 1:|\psi\rangle_{4}^{\mathrm{A} 1}=\sqrt{c}|\mathrm{GHZ}\rangle_{4}+\mathrm{e}^{i \varphi} \sqrt{1-c}|\psi\rangle_{4}, \\
& \mathrm{~A} 2:|\psi\rangle_{4}^{\mathrm{A} 2}=\sqrt{c}|\mathrm{GHZ}\rangle_{4}+\mathrm{e}^{i \varphi} \sqrt{1-c}\left|\mathrm{GHZ}^{\prime}\right\rangle_{4}, \\
& \mathrm{~A} 3:|\psi\rangle_{4}^{\mathrm{A} 3}=\sqrt{c}|W\rangle_{4}+\mathrm{e}^{i \varphi} \sqrt{1-c}|\psi\rangle_{4}, \\
& \mathrm{~A} 4:|\psi\rangle_{4}^{\mathrm{A} 4}=\sqrt{c}|W\rangle_{4}+\mathrm{e}^{i \varphi} \sqrt{1-c}\left|\mathrm{GHZ}^{\prime}\right\rangle_{4}, \\
& \mathrm{~A} 5:|\psi\rangle_{4}^{\mathrm{A} 5}=\sqrt{c}\left|\mathrm{GHZ}^{\prime}\right\rangle_{4}+\mathrm{e}^{i \varphi} \sqrt{1-c}|\psi\rangle_{4},
\end{aligned}
$$

where $c \in[0,1]$ and $\varphi \in[0,2 \pi) . \varphi$ is set to be $\pi / 3$ for all numerical evaluations below.

In order to highlight the effect of TIs in HTIEs, $c$ is set to be three different values in this section, $c=$ $1 / 4,1 / 2,3 / 4$, which are labeled as $\mathrm{A} N-1, \mathrm{~A} N-2$ and $\mathrm{A} N-$ 3 , as shown in Table П. $N=1,2,3,4,5$ denote the different HTIEs in Eq.(154), and the letter A is used to distinguish them from the other multi-qubit cases. Similar notations are also applied for the remainder of this section. The different choices for the values of $c$ are to obtain a general conclusion that is independent on the special superposition coefficients. The notations case 1 


\begin{tabular}{llllr}
\hline \hline State & Case 0 & Case 1 & Case 2 & Case 3 \\
\hline A1-1 & 0.28253 & - & 0.29458 & $\mathbf{0 . 2 9 5 3}$ \\
A1-2 & 0.28452 & - & 0.29077 & $\mathbf{0 . 2 9 1 6}$ \\
A1-3 & 0.34184 & - & 0.37407 & $\mathbf{3 / 8 = 0 . 3 7 5}$ \\
\hline A2-1 & 0.3495 & - & $\mathbf{3 / 8 = 0 . 3 7 5}$ & 0.18 \\
A2-2 & 0.24912 & - & $\mathbf{0 . 2 5}$ & $\mathbf{0 . 2 5}$ \\
A2-3 & 0.33599 & - & 0.37282 & $\mathbf{3 / 8 = 0 . 3 7 5}$ \\
\hline A3-1 & 0.47811 & - & 0.50217 & $\mathbf{0 . 5 0 3 6 4}$ \\
A3-2 & 0.51147 & - & 0.57522 & $\mathbf{0 . 5 7 8 2}$ \\
A3-3 & 0.52825 & - & 0.58671 & $\mathbf{0 . 5 8 8 3 2}$ \\
\hline A4-1 & 0.44235 & - & $\mathbf{0 . 4 9 0 3 7}$ & 0.35 \\
A4-2 & 0.43111 & - & $\mathbf{0 . 4 8 2 0 1}$ & 0.444 \\
A4-3 & 0.47361 & - & 0.49538 & $\mathbf{0 . 4 9 5 8 0}$ \\
\hline A5-1 & 0.29404 & 0.29524 & 0.29518 & $\mathbf{0 . 2 9 5 3 0}$ \\
A5-2 & 0.28207 & $\mathbf{0 . 2 9 1 5 7}$ & 0.27564 & 0.27589 \\
A5-3 & 0.36237 & $\mathbf{3 / 8 = 0 . 3 7 5 0 . 2 3 2 7 4}$ & 0.2328 \\
\hline \hline
\end{tabular}

TABLE I: Numerical results of the maximal overlap for fourqubit mixed TI entangled states defined in Eqs.(54) with $\varphi=$ $\frac{\pi}{3}$.

and case 2 in Table I respectively denote the two suppositions for the nearest FSPs, based on the TIs of the first and second term in HTIEs Eq. (54). For example, for $|\psi\rangle_{4}^{\mathrm{A} 5}$ case 1 means the supposition for the nearest FSPs $a_{1}=a_{3}, a_{2}=a_{4}$ and $\theta_{1}=\theta_{3}, \theta_{2}=\theta_{4}$, based on the two-period TI of $\left|\mathrm{GHZ}^{\prime}\right\rangle_{4}$, while case 2 means the supposition $a_{1}=a_{2}, a_{3}=a_{4}$ and $\theta_{1}=\theta_{2}, \theta_{3}=\theta_{4}$, based on the four-period TI of $|\psi\rangle_{4}$.

The numerical results are shown in Table [1. Interestingly a hierarchy for the basic TI entangled states can be found. For the state $|\psi\rangle_{4}^{\mathrm{A} 1(3)}$, the maximal overlap occurs with the supposition of permutation invariance for the nearest FSPs. This feature is not surprising because the nearest FSPs for $|\psi\rangle_{4}$ may be permutationally invariant, as shown in Sec IIIB. In contrast, the maximal overlap for $|\psi\rangle_{4}^{\mathrm{A} 5}$ can be attained only for FSPs decided by $\left|\mathrm{GHZ}^{\prime}\right\rangle_{4}$, as shown by A5-2 and A5-3 in Table \. As for $\mathrm{A} 5-1$, the difference between case 1 and case 3 is just of order $10^{-5}$, which can be seen as the numerical error. This observation implies that the TI structure of $\left|\mathrm{GHZ}^{\prime}\right\rangle_{4}$ is predominant for the determination of $\Lambda_{\max }$ for $|\psi\rangle_{4}^{\mathrm{A} 5}$, and thus we say $\left|\mathrm{GHZ}^{\prime}\right\rangle_{4}$ has a higher order of symmetry than $|\psi\rangle_{4}$, i.e. $\mathcal{S}_{\left|\mathrm{GHZ}^{\prime}\right\rangle_{4}}>\mathcal{S}_{|\psi\rangle_{4}}$. For $|\psi\rangle_{4}^{\mathrm{A} 2}$, the numerical results for $\mathrm{A} 2$ in Table 1 show that $\Lambda_{\max }$ depends only on the superposition coefficients, which imply that $|\mathrm{GHZ}\rangle_{4}$ and $\left|\mathrm{GHZ}^{\prime}\right\rangle_{4}$ have the same order of symmetry, $\mathcal{S}_{|\mathrm{GHZ}\rangle_{4}} \sim \mathcal{S}_{\left|\mathrm{GHZ}^{\prime}\right\rangle_{4}}$. As for A4, one can obtain the order $\mathcal{S}_{\left|\mathrm{GHZ}^{\prime}\right\rangle_{4}}>\mathcal{S}_{|W\rangle}$.

It is intricate for the relation of $|W\rangle$ and $|\psi\rangle_{4}$; only by A3 in Table I we cannot tell which has a higher order of symmetry because both of the nearest FSPs for $|W\rangle$ and $|\psi\rangle_{4}$ are permutationally invariant. However, we should point out that $|\psi\rangle_{4}$ actually is biseparable, which is the main reason for the existence of the PI nearest FSPs.

\begin{tabular}{llllr}
\hline \hline State & Case 0 & Case 1 & Case 2 & Case 3 \\
\hline B1-1 & 0.20702 & - & 0.24871 & $\mathbf{0 . 2 5 1 2 4}$ \\
B1-2 & 0.24403 & - & 0.27173 & $\mathbf{0 . 2 7 3 2 9}$ \\
B1-3 & 0.31523 & - & 0.37251 & $\mathbf{3 / 8 = 0 . 3 7 5}$ \\
\hline B2-1 & 0.33628 & - & 0.40932 & $\mathbf{0 . 4 1 0 2}$ \\
B2-2 & 0.44841 & - & 0.49339 & $\mathbf{0 . 4 9 5 9 7}$ \\
B2-3 & 0.39626 & - & 0.52329 & $\mathbf{0 . 5 2 7 3 4}$ \\
\hline B3-1 & 0.22777 & 0.24746 & 0.24749 & $\mathbf{0 . 2 4 7 6 3}$ \\
B3-2 & 0.22326 & 0.25915 & 0.25899 & $\mathbf{0 . 2 5 9 2 0}$ \\
B3-3 & 0.22985 & 0.24752 & 0.24707 & $\mathbf{0 . 2 4 7 6 2}$ \\
\hline \hline
\end{tabular}

TABLE II: Numerical results of the maximal overlap for fivequbit mixed TI entangled states defined in Eqs. (56) with $\varphi=$ $\frac{\pi}{3}$.

Thus we set $\mathcal{S}_{|W\rangle_{4}}>\mathcal{S}_{|\psi\rangle_{4}}$.

In conclusion, one has

$$
\mathcal{S}_{|\mathrm{GHZ}\rangle_{4}} \sim \mathcal{S}_{\left|\mathrm{GHZ}^{\prime}\right\rangle_{4}}>\mathcal{S}_{|W\rangle_{4}}>\mathcal{S}_{|\psi\rangle_{4}}
$$

\section{5-qubit case}

This situation is simple because 5 is prime number, and there are only one- and five-period TI entangled states. Then the HTIEs are

$$
\begin{aligned}
& \mathrm{B} 1:|\psi\rangle_{5}^{\mathrm{B} 1}=\sqrt{c}|\mathrm{GHZ}\rangle_{5}+\mathrm{e}^{i \varphi} \sqrt{1-c}\left|\psi^{1 a}\right\rangle_{5}, \\
& \mathrm{~B} 2:|\psi\rangle_{5}^{\mathrm{B} 2}=\sqrt{c}|W\rangle_{5}+\mathrm{e}^{i \varphi} \sqrt{1-c}\left|\psi^{1 a}\right\rangle_{5} \\
& \mathrm{~B} 3:|\psi\rangle_{5}^{\mathrm{B} 3}=\sqrt{c} \sqrt{1-c}\left|\psi^{1 a}\right\rangle_{5}+\mathrm{e}^{i \varphi} \sqrt{1-c}\left|\psi^{1 b}\right\rangle_{5}(5 c
\end{aligned}
$$

As shown in Table III the optimal fully separable states can be obtained always under the supposition of PI. Thus one has the order of symmetry $\mathcal{S}_{|\mathrm{GHZ}\rangle_{5}}>\mathcal{S}_{\left|\psi^{1 a}\right\rangle_{5}}$ and $\mathcal{S}_{|W\rangle_{5}}>\mathcal{S}_{\left|\psi^{1 a}\right\rangle_{5}}$. As for $|W\rangle_{5}$ and $|\mathrm{GHZ}\rangle_{5}$, we could temporarily set $\mathcal{S}_{|\mathrm{GHZ}\rangle_{5}}>\mathcal{S}_{|W\rangle_{5}}$ because $|\mathrm{GHZ}\rangle_{5}$ is oneperiod, while $|W\rangle_{5}$ is five-period. A strange result of B3 is that the optimal FSPs is permutationally invariant, adn independent on the TI structures of components in $|\psi\rangle_{5}^{\mathrm{B} 3}$. Thus $\left|\psi^{1 a}\right\rangle_{5}$ and $\left|\psi^{1 b}\right\rangle_{5}$ could be considered same order.

In total one has a hierarchy of the symmetry, in this case

$$
\mathcal{S}_{|\mathrm{GHZ}\rangle_{5}}>\mathcal{S}_{|W\rangle_{5}}>\mathcal{S}_{\left|\psi^{1 a}\right\rangle_{5}} \sim \mathcal{S}_{\left|\psi^{1 b}\right\rangle_{5}}
$$

\section{6-qubit case}

This case is more complicated because there are four types of basic TI entangled states. Thus the discussion focuses on the superposition of basic TI entangled states 


\begin{tabular}{|c|c|c|c|c|}
\hline State & Case 0 & Case 1 & Case 2 & Case 3 \\
\hline C1-1 & 0.24839 & - & \multicolumn{2}{|c|}{$\mathbf{3} / \mathbf{8}=\mathbf{0 . 3 7 5} 1 / 8=0.125$} \\
\hline C1-2 & 0.18059 & - & 0.25 & 0.25 \\
\hline $\mathrm{C} 1-3$ & 0.25466 & - & 0.37159 & $3 / 8=0.375$ \\
\hline $\mathrm{C} 2-1$ & 0.29698 & - & $3 / 8=0.375$ & 0.159 \\
\hline $\mathrm{C} 2-2$ & 0.21546 & - & 0.2672 & 0.25793 \\
\hline $\mathrm{C} 2-3$ & 0.25309 & - & 0.34657 & 0.34658 \\
\hline C3-1 & 0.16963 & - & 0.1952 & 0.20225 \\
\hline C3-2 & 0.20256 & - & 0.24579 & 0.25706 \\
\hline C3-3 & 0.2557 & - & 0.35873 & $3 / 8=0.375$ \\
\hline C4-1 & 0.24022 & - & 0.3537 & 0.35595 \\
\hline C4-2 & 0.30666 & - & 0.44431 & 0.44687 \\
\hline $\mathrm{C} 4-3$ & 0.33181 & - & 0.48512 & 0.48992 \\
\hline C5-1 & 0.11394 & - & 0.12491 & $1 / 8=0.125$ \\
\hline C5-2 & 0.19292 & - & 0.24775 & 0.25 \\
\hline C5-3 & 0.2892 & - & 0.37285 & $3 / 8=0.375$ \\
\hline C6-1 & 0.17516 & - & 0.22896 & 0.22998 \\
\hline C6-2 & 0.20439 & - & 0.31553 & 0.31641 \\
\hline C6-3 & 0.24171 & - & 0.38406 & 0.38689 \\
\hline $\mathrm{C} 7-1$ & 0.1474 & 0.15244 & 0.15161 & 0.153 \\
\hline $\mathrm{C} 7-2$ & 0.15756 & 0.25 & 0.1344 & 0.135 \\
\hline $\mathrm{C} 7-3$ & 0.2534 & \multicolumn{2}{|c|}{$\mathbf{3} / \mathbf{8}=\mathbf{0 . 3 7 5 0 . 1 0 2 1 1}$} & 0.10273 \\
\hline $\mathrm{C} 8-1$ & 0.11912 & \multicolumn{2}{|c|}{$\mathbf{1} / \mathbf{8}=\mathbf{0 . 1 2 5 0 . 1 2 4 5 6}$} & 0.1015 \\
\hline $\mathrm{C} 8-2$ & 0.21251 & 0.25 & 0.08947 & 0.09 \\
\hline $\mathrm{C} 8-3$ & 0.27651 & \multicolumn{2}{|c|}{$\mathbf{3} / \mathbf{8}=\mathbf{0 . 3 7 5 0 . 0 7 0 2 5}$} & 0.07 \\
\hline C9-1 & 0.19456 & - & 0.25 & 0.14 \\
\hline C9-2 & 0.19164 & - & 0.24937 & 0.25 \\
\hline $\mathrm{C} 9-3$ & 0.27267 & - & 0.37207 & $3 / 8=0.375$ \\
\hline C10-1 & 0.25579 & - & 0.33068 & 0.2603 \\
\hline $\mathrm{C} 10-2$ & 0.26902 & - & 0.36098 & 0.36095 \\
\hline $\mathrm{C} 10-3$ & 0.26567 & - & 0.42781 & 0.42782 \\
\hline $\mathrm{C} 11-1$ & 0.18722 & 0.12411 & 0.25 & 0.09 \\
\hline $\mathrm{C} 11-2$ & 0.18246 & 0.25 & 0.16624 & 0.085 \\
\hline $\mathrm{C} 11-3$ & 0.25725 & \multicolumn{2}{|c|}{$\mathbf{3} / \mathbf{8}=\mathbf{0 . 3 7 5 0 . 0 8 2 8 6}$} & 0.072 \\
\hline $\mathrm{C} 12-1$ & 0.13548 & 0.15535 & 0.15547 & 0.15555 \\
\hline $\mathrm{C} 12-2$ & 0.13392 & \multicolumn{2}{|c|}{$\mathbf{1 / 6} \approx \mathbf{0 . 1 6 6} 7.14518$} & 0.14532 \\
\hline $\mathrm{C} 12-3$ & 0.20826 & 0.25 & 0.12247 & 0.12263 \\
\hline $\mathrm{C} 13-1$ & 0.12497 & 0.14731 & 0.14731 & 0.14731 \\
\hline $\mathrm{C} 13-2$ & 0.11305 & \multicolumn{2}{|c|}{$\mathbf{1 / 6} \approx \mathbf{0 . 1 6 6 7 . 1 4 5 4 2}$} & 0.14542 \\
\hline C13-3 & 0.15592 & 0.25 & 0.12959 & 0.12960 \\
\hline
\end{tabular}

TABLE III: Numerical results of the maximal overlap for sixqubit mixed TI entangled states defined in Eqs.(58) with $\varphi=$ $\frac{\pi}{3}$. with different periodic structures,

$$
\begin{aligned}
\mathrm{C} 1:|\psi\rangle_{6}^{\mathrm{C} 1} & =\sqrt{c}|\mathrm{GHZ}\rangle_{6}+\mathrm{e}^{i \varphi} \sqrt{1-c}\left|\mathrm{GHZ}^{\prime}\right\rangle_{6}, \\
\mathrm{C} 2:|\psi\rangle_{6}^{\mathrm{C} 2} & =\sqrt{c}|W\rangle_{6}+\mathrm{e}^{i \varphi} \sqrt{1-c}\left|\mathrm{GHZ}^{\prime}\right\rangle_{6}, \\
\mathrm{C} 3:|\psi\rangle_{6}^{\mathrm{C} 3} & =\sqrt{c}|\mathrm{GHZ}\rangle_{6}+\mathrm{e}^{i \varphi} \sqrt{1-c}\left|\psi^{1 a}\right\rangle_{6}, \\
\mathrm{C} 4:|\psi\rangle_{6}^{\mathrm{C} 4} & =\sqrt{c}|W\rangle_{6}+\mathrm{e}^{i \varphi} \sqrt{1-c}\left|\psi^{1 a}\right\rangle_{6}, \\
\mathrm{C} 5:|\psi\rangle_{6}^{\mathrm{C} 5} & =\sqrt{c}|\mathrm{GHZ}\rangle_{6}+\mathrm{e}^{i \varphi} \sqrt{1-c}\left|\psi^{2 a}\right\rangle_{6}, \\
\mathrm{C} 6:|\psi\rangle_{6}^{\mathrm{C} 6} & =\sqrt{c}|W\rangle_{6}+\mathrm{e}^{i \varphi} \sqrt{1-c}\left|\psi^{2 a}\right\rangle_{6}, \\
\mathrm{C} 7:|\psi\rangle_{6}^{\mathrm{C} 7} & =\sqrt{c}\left|\mathrm{GHZ}^{\prime}\right\rangle_{6}+\mathrm{e}^{i \varphi} \sqrt{1-c}\left|\psi^{1 a}\right\rangle_{6}, \\
\mathrm{C} 8:|\psi\rangle_{6}^{\mathrm{C} 8} & =\sqrt{c}\left|\mathrm{GHZ}^{\prime}\right\rangle_{6}+\mathrm{e}^{i \varphi} \sqrt{1-c}\left|\psi^{2 a}\right\rangle_{6}, \\
\mathrm{C} 9:|\psi\rangle_{6}^{\mathrm{C} 9} & \left.=\sqrt{c}\left|\mathrm{GHZ}_{6}+\mathrm{e}^{i \varphi} \sqrt{1-c}\right| \psi^{3}\right\rangle_{6}, \\
\mathrm{C} 10:|\psi\rangle_{6}^{\mathrm{C} 10} & =\sqrt{c}|W\rangle_{6}+\mathrm{e}^{i \varphi} \sqrt{1-c}\left|\psi^{3}\right\rangle_{6}, \\
\mathrm{C} 11:|\psi\rangle_{6}^{\mathrm{C} 11} & =\sqrt{c}\left|\mathrm{GHZ}^{\prime}\right\rangle_{6}+\mathrm{e}^{i \varphi} \sqrt{1-c}\left|\psi^{3}\right\rangle_{6}, \\
\mathrm{C} 12:|\psi\rangle_{6}^{\mathrm{C} 12} & =\sqrt{c}\left|\psi^{3}\right\rangle_{6}+\mathrm{e}^{i \varphi} \sqrt{1-c}\left|\psi^{1 a}\right\rangle_{6}, \\
\mathrm{C} 13:|\psi\rangle_{6}^{\mathrm{C} 13} & =\sqrt{c}\left|\psi^{3}\right\rangle_{6}+\mathrm{e}^{i \varphi} \sqrt{1-c}\left|\psi^{2 a}\right\rangle_{6} .
\end{aligned}
$$

$\left|\psi^{1 a}\right\rangle_{6}$ and $\left|\psi^{1 b}\right\rangle_{6}$ are considered equivalent numerically because the only difference is the position of single-party state $|1\rangle_{\mathrm{s}}$, therefore $\left|\psi^{2 a(b, c)}\right\rangle_{6}$.

The numerical results are listed in Table III. Some features similar to those of the four-qubit case can be found. It is obvious from $\mathrm{C} 1$ in Table IIII that $\Lambda_{\max }$ depends only on superposition coefficients, and thus $\mathcal{S}_{|\mathrm{GHZ}\rangle_{6}} \sim \mathcal{S}_{\left|\mathrm{GHZ}^{\prime}\right\rangle_{6}}$. For C2, the supposition for the nearest fully separable state is optimal from the TI structure of $\left|\mathrm{GHZ}^{\prime}\right\rangle_{6}$, and thus $\mathcal{S}_{\left|\mathrm{GHZ}^{\prime}\right\rangle_{6}}>\mathcal{S}_{|W\rangle_{6}}$. By C3, C4, C5, C6, C7, and C8 in Table III, $\left|\psi^{1 a}\right\rangle_{6}$ and $\left|\psi^{2 a}\right\rangle_{6}$ both show lower order of the symmetry than $|\mathrm{GHZ}\rangle_{6},\left|\mathrm{GHZ}^{\prime}\right\rangle_{6}$ and $|W\rangle_{6}$ because $\Lambda_{\max }$ is decided mainly by the TI of the latter.

A particular state in this case is $\left|\psi^{3}\right\rangle_{6}$, which is threeperiod. By C9 and C11 in Table III $\Lambda_{\max }$ depends only on the superposition coefficients. However, because $\left|\psi^{3}\right\rangle_{6}$ is three-period, thus $\mathcal{S}_{\left|\psi^{3}\right\rangle_{6}}<\mathcal{S}_{|\mathrm{GHZ}\rangle_{6}} \sim \mathcal{S}_{\left|\mathrm{GHZ}^{\prime}\right\rangle_{6}}$, whereas from $\mathrm{C} 10, \mathrm{C} 12$, and $\mathrm{C} 13,\left|\psi^{3}\right\rangle_{6}$ always has a noticeable effect on $\Lambda_{\max }$.

In conclusion, one obtains

$$
\mathcal{S}_{|\mathrm{GHZ}\rangle_{6}} \sim \mathcal{S}_{\left|\mathrm{GHZ}^{\prime}\right\rangle_{6}}>\mathcal{S}_{\left|\psi^{3}\right\rangle_{6}}>\mathcal{S}_{|W\rangle_{6}}>\mathcal{S}_{\left|\psi^{1(2) a}\right\rangle_{6}}
$$

It is exceptional for the relation of $\left|\psi^{1 a}\right\rangle_{6}$ and $\left|\psi^{2 a}\right\rangle_{6}$, as will shown at the end of this section. 


\begin{tabular}{llllr}
\hline \hline State & Case 0 & Case 1 & Case 2 & Case 3 \\
\hline D1-1 & 0.17958 & - & $\mathbf{3} / \mathbf{8}=\mathbf{0 . 3 7 5}$ & $1 / 8=0.125$ \\
D1-2 & 0.11301 & - & $\mathbf{0 . 2 5}$ & $\mathbf{0 . 2 5}$ \\
D1-3 & 0.16164 & - & 0.37167 & $\mathbf{3} / \mathbf{8}=\mathbf{0 . 3 7 5}$ \\
\hline D2-1 & 0.16865 & - & $\mathbf{3 / 8 = 0 . 3 7 5}$ & 0.12 \\
D2-2 & 0.1515 & - & $\mathbf{0 . 2 5}$ & 0.20792 \\
D2-3 & 0.14598 & - & $\mathbf{0 . 3 0 4 1 5}$ & $\mathbf{0 . 3 0 4 1 6}$ \\
\hline D3-1 & 0.09073 & - & $\mathbf{3 / 1 6 = 0 . 1 8 7 5}$ & 0.1307 \\
D3-2 & 0.12702 & - & 0.24895 & $\mathbf{0 . 2 5}$ \\
D3-3 & 0.14792 & - & 0.37068 & $\mathbf{3} / \mathbf{8}=\mathbf{0 . 3 7 5}$ \\
\hline D4-1 & 0.12409 & - & $\mathbf{0 . 2 4 9 2 4}$ & 0.22288 \\
D4-2 & 0.17166 & - & $\mathbf{0 . 3 2 3 7 3}$ & $\mathbf{0 . 3 2 3 7 3}$ \\
D4-3 & 0.15625 & - & $\mathbf{0 . 3 9 6 5 6}$ & $\mathbf{0 . 3 9 6 5 6}$ \\
\hline D5-1 & 0.08002 & - & $\mathbf{3 / 1 6 = 0 . 1 8 7 5} / 8=0.125$ \\
D5-2 & 0.13844 & - & 0.24804 & $\mathbf{0 . 2 5}$ \\
D5-3 & 0.14722 & - & 0.3733 & $\mathbf{3} / \mathbf{8}=\mathbf{0 . 3 7 5}$ \\
\hline D6-1 & 0.08814 & - & $\mathbf{3 / 1 6} \approx \mathbf{0 . 1 8 7 0}$ & $\mathbf{3} 8511464$ \\
D6-2 & 0.09481 & - & $\mathbf{0 . 2 1 3 3 8}$ & $\mathbf{0 . 2 1 3 3 7}$ \\
D6-3 & 0.15491 & - & 0.30844 & $\mathbf{0 . 3 0 8 4 6}$ \\
\hline D7-1 & 0.11729 & 0.13375 & $\mathbf{3 / 1 6 = 0 . 1 8 7 5}$ & 0.04402 \\
D7-2 & 0.11472 & $\mathbf{0 . 2 5}$ & 0.12349 & 0.03601 \\
D7-3 & 0.2053 & $\mathbf{3 / 8 = 0 . 3 7 5 0 . 0 6 2 1 9}$ & 0.02544 \\
\hline D8-1 & 0.0962 & 0.12346 & $\mathbf{3 / 1 6 = 0 . 1 8 7 5}$ & 0.1015 \\
D8-2 & 0.13414 & $\mathbf{0 . 2 5}$ & 0.12402 & 0.017 \\
D8-3 & 0.17557 & $\mathbf{3 / 8 = 0 . 3 7 5 0 . 0 6 2 0 4}$ & 0.01455 \\
\hline D9-1 & 0.07897 & $\mathbf{3 / 1 6 = 0 . 1 8 7 . 5 6 4 2 4}$ & 0.3486 \\
D9-2 & 0.07395 & $\mathbf{1 / 8}$ & $\mathbf{1 / 8}$ & 0.04389 \\
D9-3 & 0.09303 & 0.0258 & $\mathbf{3 / 1 6 = 0 . 1 8 7 5}$ & 0.04943 \\
\hline \hline & & & & \\
\hline
\end{tabular}

TABLE IV: Numerical results of the maximal overlap for eight-qubit mixed TI entangled states defined in Eqs. (60).

\section{E. 8-qubit case}

Eight-qubit HTIEs are studied in order to display the generality of the hierarchy for basic TI entangled states,

$$
\begin{aligned}
& \text { D1: }|\psi\rangle_{6}^{\mathrm{D} 1}=\sqrt{c}|\mathrm{GHZ}\rangle_{8}+\mathrm{e}^{i \varphi} \sqrt{1-c}\left|\mathrm{GHZ}^{\prime}\right\rangle_{8}, \\
& \text { D2: }|\psi\rangle_{6}^{\mathrm{D} 2}=\sqrt{c}|W\rangle_{8}+\mathrm{e}^{i \varphi} \sqrt{1-c}\left|\mathrm{GHZ}^{\prime}\right\rangle_{8}, \\
& \text { D3: }|\psi\rangle_{6}^{\mathrm{D} 3}=\sqrt{c}|\mathrm{GHZ}\rangle_{8}+\mathrm{e}^{i \varphi} \sqrt{1-c}\left|\psi^{1}\right\rangle_{8}, \\
& \text { D4: }|\psi\rangle_{6}^{\mathrm{D} 4}=\sqrt{c}|W\rangle_{8}+\mathrm{e}^{i \varphi} \sqrt{1-c}\left|\psi^{1}\right\rangle_{8}, \\
& \text { D5: }|\psi\rangle_{6}^{\mathrm{D} 5}=\sqrt{c}|\mathrm{GHZ}\rangle_{8}+\mathrm{e}^{i \varphi} \sqrt{1-c}\left|\psi^{2}\right\rangle_{8}, \\
& \text { D6: }|\psi\rangle_{6}^{\mathrm{D} 6}=\sqrt{c}|W\rangle_{8}+\mathrm{e}^{i \varphi} \sqrt{1-c}\left|\psi^{2}\right\rangle_{8}, \\
& \text { D7: }|\psi\rangle_{6}^{\mathrm{D} 7}=\sqrt{c}\left|\mathrm{GHZ}^{\prime}\right\rangle_{8}+\mathrm{e}^{i \varphi} \sqrt{1-c}\left|\psi^{1}\right\rangle_{8}, \\
& \text { D8: }|\psi\rangle_{6}^{\mathrm{D} 8}=\sqrt{c}\left|\mathrm{GHZ}^{\prime}\right\rangle_{8}+\mathrm{e}^{i \varphi} \sqrt{1-c}\left|\psi^{2}\right\rangle_{8}, \\
& \text { D9: }|\psi\rangle_{6}^{\mathrm{D} 9}=\sqrt{c}\left|\psi^{1}\right\rangle_{8}+\mathrm{e}^{i \varphi} \sqrt{1-c}\left|\psi^{2}\right\rangle_{8} .
\end{aligned}
$$

From Table IV features similar to those of four- and six-qubit cases can be found. For D1 and D9, $\Lambda_{\max }$ is obviously dependent only on the superposition coefficients of $|\psi\rangle_{6}^{\mathrm{D} 1}$ and $|\psi\rangle_{6}^{\mathrm{D} 9}$, and thus $\left.\mathcal{S}_{|\mathrm{GHZ}\rangle_{8}} \sim \mathcal{S}_{\mid \mathrm{GHZ}}\right\rangle_{8}$

\begin{tabular}{llllr}
\hline \hline State & Case 0 & Case 1 & Case 2 & Case 3 \\
\hline E1-1 & $\mathbf{0 . 2 4 0 7}$ & 0.18365 & 0.20275 & 0.18365 \\
E1-2 & $\mathbf{0 . 2 5 1 9 9}$ & 0.20546 & 0.21439 & 0.20545 \\
E1-3 & $\mathbf{0 . 2 4 2 0 9}$ & 0.20251 & 0.20574 & 0.20251 \\
\hline \hline
\end{tabular}

TABLE V: Numerical results of the maximal overlap for the mixed TI entangled states Eqs. (62). The data for case 0 are obtained by setting $\theta_{1}=\theta_{2}=\cdots=\theta_{6}=-\varphi$

and $\mathcal{S}_{\left|\psi^{1}\right\rangle_{8}} \sim \mathcal{S}_{\left|\psi^{2}\right\rangle_{8}}$. As for D3, D5, D7, and D8, $\Lambda_{\max }$ is also dependent only on the superposition coefficients. However, because the common coefficient of $\left|\psi^{1(2)}\right\rangle_{8}$ is $1 / 2$, which is smaller than that of $|\mathrm{GHZ}\rangle_{8}$ and $\left|\mathrm{GHZ}^{\prime}\right\rangle_{8}$, then $\mathcal{S}_{|\mathrm{GHZ}\rangle_{8}} \sim \mathcal{S}_{\left|\mathrm{GHZ}^{\prime}\right\rangle_{8}}>\mathcal{S}_{\left|\psi^{1(2)}\right\rangle_{8}}$. From D2, D4 and D6, $\Lambda_{\max }$ can always be obtained under the supposition for nearest FSPs based on the TI structures of $\left|\mathrm{GHZ}^{\prime}\right\rangle_{8}$ or $\left|\psi^{1(2)}\right\rangle_{8}$, and thus $\mathcal{S}_{|W\rangle_{8}}<\mathcal{S}_{\left|\mathrm{GHZ}^{\prime}\right\rangle_{8}}$ and $\mathcal{S}_{|W\rangle_{8}}<\mathcal{S}_{\left|\psi^{1(2)}\right\rangle_{8}}$.

In total one has

$$
\mathcal{S}_{|\mathrm{GHZ}\rangle_{8}} \sim \mathcal{S}_{\left|\mathrm{GHZ}^{\prime}\right\rangle_{8}}>\mathcal{S}_{\left|\psi^{1}\right\rangle_{8}} \sim \mathcal{S}_{\left|\psi^{2}\right\rangle_{8}}>\mathcal{S}_{|W\rangle_{8}} .
$$

\section{F. Further discussion}

In conclusion, the hierarchy for the basic TI entangled states is a general feature for multi-qubit states. An important observation obtained from the studies discussed above is that this hierarchy has an important effect on the determination of $\Lambda_{\max }$ of HTIEs.

From the above numerical evaluations, two observations can be made

1. $\Lambda_{\max }$ for the HTIEs, composed of two different basic TI entangled states except of the $N$-period, depends obviously on the superposition coefficients. That is to say, $\Lambda_{\max }$ are just the maximal one of the superposition coefficients times the common coefficient of the basic TI entangled states. For example, as shown for A2 in Table I. $\Lambda_{\max }$ are $\left(1-\frac{1}{4}\right) \times \frac{1}{2}$, $\frac{1}{2} \times \frac{1}{2}, \frac{3}{4} \times \frac{1}{2}$ for $c=1 / 4,1 / 2$ and $c=3 / 4$, respectively. More examples can be found in $\mathrm{C} 1, \mathrm{C} 9$, and C11 in Table II and D1, D3, D5, D7, D8, and D9 in Table IV This feature is consistent with the result of Ref. [28].

2. The situation for the cases including $N$-period TI entangled states is very complex, however. A general feature is that $\Lambda_{\max }$ in this case is unrelated completely to the superposition coefficients. However, one can find that PI is an optimal supposition for the nearest FSPs when the HTIEs are composed of one- and $N$-period TI entangled states, independent on the coefficient 27]. For other cases our data cannot give a clear conclusion.

A unique case is one of the HTIEs composed of basic $N$-period TI entangled states, except of $|W\rangle_{N}$. For 
example, let us consider HTIEs,

$$
|\psi\rangle_{6}^{E 1}=\sqrt{c}\left|\psi^{1 a}\right\rangle_{6}+\mathrm{e}^{i \varphi}\left|\psi^{2 a}\right\rangle_{6},
$$

of which $\Lambda_{\max }$ are listed in Table $\mathrm{V}$ It is obvious that $\Lambda_{\max }$ is completely independent of the TI structures of $\left|\psi^{1 a}\right\rangle_{6}$ and $\left|\psi^{2 a}\right\rangle_{6}$. A similar feature can be found also for the eight-qubit case, while our evaluation show that this feature does not appear for D9 in Table IV] which is the superposition of two four-period TI entangled states for eight-qubit multipartite states. The above evaluations show that this feature does not occur for the combination with different periods. So we surmise that it would be unique for $N$-period basic TI entangled states, except for $|W\rangle_{N}$, and one has to be careful when dealing with this case.

Unfortunately we cannot provide the exact proofs for these observations above, and the underlying physics is also unclear until now. However, we should express the idea here that it would be efficient to find the nearest fully separable state from the TI of entangled states.

Here we discuss the numerical errors of the random simulation of $\Lambda_{\max }$. The numerical evaluation for $\Lambda_{\max }$ is actually an exhaustive method: sampling randomly the permissible values for all independent parameters as much as possible and record the maximal value of the overlap. It is obvious that the efficiency and accuracy of this algorithm is dependent on the sampling times and the number of independent parameters: greater sampling times help us achieve the true result more accurately, but the evaluation time increase. The more independent parameters we use, the slower is the convergence of evaluation to the true result. Because the purpose of this section is to show the effect of TI rather than to find the true $\Lambda_{\max }$, we chose sampling times of $10^{5}$ in order for a tolerable calculation time, and $\Lambda_{\max }$ is the steady numerical result that is unchanged for $10^{4}$ sampling times. We admit that the evaluation in this section is crude. However some interesting information can still be attained from the data listed in the previous tables. In addition, the number of independent parameters can be reduced by some algebra, e.g., Eq. (18) which makes our evaluation more reliable.

\section{SUMMARY AND CONCLUSION}

By several exact and numerical examples, we presents a comprehensive study of the maximal overlap with fully separable state for the multipartite entangled pure state with translational invariance. As shown in the above discussions, translational invariance of the entangled state has an intrinsic effect on the determination of the maximal overlap $\Lambda_{\max }$ and the nearest fully separable state.

One contribution of this article is the introduction of the basic TI entangled states, as shown in Sec. III] The key point for these states is that the other types of TI entangled states can be expressed as the superposition of these basic ones. In addition for these basic TI entangled states $\Lambda_{\max }$ and the nearest fully separable state can be determined just by utilizing the TI. Furthermore, we stress that TI or basic TI entangled states actually defines a connectedness in the states belonging to different parties, and this connectedness is the unique fundamental character that is distinct from one state to the other state. So the nearest fully separable state for the entangled state should also manifest this unique TI.

Another contribution of this paper is the demonstration of the hierarchy for the basic TI entangled states with different periodic structure. This hierarchy of TI has a fundamental effect on the evaluation of $\Lambda_{\max }$ for HTIEs, as shown in Sec. V (see Sec. VF for a conclusion).

Finally, we have to admit that an exact proof for the resultant conclusions are still absent. From our point of view it is because the understanding of multipartite entanglement is not yet unified. As shown in this paper, although the forms of the multipartite entanglements are diverse, there are some fundamental features by which one can obtain useful information, such as the introduction of basic TI entangled states and their hierarchy. So it is promising to understand multipartite entanglement by disclosing these fundamental features.

\section{Acknowledgments}

One of the authors (H.T.C) thanks Prof. Dr. Xue-Xi Yi for his hospitality during his visitto Dalian University of Technology, where the work was finally completed. Helpful discussion with Prof. Dr. Yan-Kui Bai and Dr. Chang-Shui $\mathrm{Yu}$, and critical readings by Dr. Lin-Cheng Wang and Dr. Xiao-Li Huang are acknowledged also. This work is supported by NSF of China, Grants No. 11005002 (Cui) and No. 11005003 (Tian).

\section{Appendix A}

This appendix provides an exact result of $\Lambda_{\max }$ at the general state,

$$
\left|W^{\prime}\right\rangle=c_{0}|100\rangle+c_{1} \mathrm{e}^{i \alpha}|010\rangle+c_{2} \mathrm{e}^{i \beta}|001\rangle,
$$

where $c_{0}, c_{1}, c_{2} \in[0,1], c_{1}^{2}+c_{2}^{2}+c_{3}^{2}=1$, and $\alpha, \beta \in[0,2 \pi]$. Then the overlap is

$$
\begin{aligned}
& \left|\left\langle W^{\prime} \mid \phi\right\rangle\right|^{2} \\
= & \mid c_{0} \sqrt{a_{1}\left(1-a_{2}\right)\left(1-a_{3}\right)} \mathrm{e}^{i\left(\theta_{2}+\theta_{3}\right)}+ \\
& c_{1} \sqrt{\left(1-a_{1}\right) a_{2}\left(1-a_{3}\right)} \mathrm{e}^{i\left(\theta_{1}+\theta_{3}-\alpha\right)}+ \\
& \left.c_{2} \sqrt{\left(1-a_{1}\right)\left(1-a_{2}\right) a_{3}} \mathrm{e}^{i\left(\theta_{1}+\theta_{2}-\beta\right)}\right|^{2} \\
\leq & {\left[c_{0} \sqrt{a_{1}\left(1-a_{2}\right)\left(1-a_{3}\right)}+c_{1} \sqrt{\left(1-a_{1}\right) a_{2}\left(1-a_{3}\right)}\right.} \\
& \left.+c_{2} \sqrt{\left(1-a_{1}\right)\left(1-a_{2}\right) a_{3}}\right]^{2}
\end{aligned}
$$


where the equality occurs when

$$
\left\{\begin{array}{l}
\theta_{2}+\theta_{3}=0 \\
\theta_{1}+\theta_{3}=\alpha \\
\theta_{1}+\theta_{2}=\beta
\end{array}\right.
$$

and the solutions can be easily obtained: $\theta_{1}=\frac{\alpha+\beta}{2}, \theta_{2}=$ $-\frac{\alpha-\beta}{2}=-\theta_{3}$.

Obviously one has

$$
\begin{gathered}
\max _{|\phi\rangle}\left|\left\langle W^{\prime} \mid \phi\right\rangle\right|^{2} \Leftrightarrow \\
\max _{a_{1}, a_{2}, a_{3}}\left[c_{0} \sqrt{a_{1}\left(1-a_{2}\right)\left(1-a_{3}\right)}+c_{1} \sqrt{\left(1-a_{1}\right) a_{2}\left(1-a_{3}\right)}\right. \\
\left.+c_{2} \sqrt{\left(1-a_{1}\right)\left(1-a_{2}\right) a_{3}}\right]^{2} .
\end{gathered}
$$

Then the following discussion would focus on the expression

$$
\begin{aligned}
f= & c_{0} \sqrt{a_{1}\left(1-a_{2}\right)\left(1-a_{3}\right)}+c_{1} \sqrt{\left(1-a_{1}\right) a_{2}\left(1-a_{3}\right)} \\
& +c_{2} \sqrt{\left(1-a_{1}\right)\left(1-a_{2}\right) a_{3}}
\end{aligned}
$$

The extremal point for $f$ corresponds to the points at which the first derivation of $f$ with $a_{1}, a_{2}, a_{3}$ vanish, i.e., $\frac{\partial f}{\partial a_{1}}=\frac{\partial f}{\partial a_{2}}=\frac{\partial f}{\partial a_{3}}=0$. This condition is transformed into the equations

$$
\left\{\begin{array}{l}
c_{1} \sqrt{\frac{a_{2}}{1-a_{2}}}+c_{2} \sqrt{\frac{a_{3}}{1-a_{3}}}=c_{0} \sqrt{\frac{1-a_{1}}{a_{1}}}, \\
c_{0} \sqrt{\frac{a_{1}}{1-a_{1}}}+c_{2} \sqrt{\frac{a_{3}}{1-a_{3}}}=c_{1} \sqrt{\frac{1-a_{2}}{a_{2}}}, \\
c_{0} \sqrt{\frac{a_{1}}{1-a_{1}}}+c_{1} \sqrt{\frac{a_{2}}{1-a_{2}}}=c_{2} \sqrt{\frac{1-a_{3}}{a_{3}}} .
\end{array}\right.
$$

Introducing the media variables $x=\sqrt{\frac{a_{1}}{1-a_{1}} \frac{a_{2}}{1-a_{2}}}, y=$ $\sqrt{\frac{a_{2}}{1-a_{2}} \frac{a_{3}}{1-a_{3}}}, z=\sqrt{\frac{a_{1}}{1-a_{1}} \frac{a_{3}}{1-a_{3}}}$, the above equation can be rewritten as

$$
\left\{\begin{array}{l}
c_{1} x+c_{2} z=c_{0}, \\
c_{0} x+c_{2} y=c_{1}, \\
c_{0} z+c_{2} y=c_{2} .
\end{array}\right.
$$

where the solutions are $x=\frac{1-2 c_{2}^{2}}{2 c_{0} c_{1}}, y=\frac{1-2 c_{0}^{2}}{2 c_{1} c_{3}}, z=\frac{1-2 c_{1}^{2}}{2 c_{0} c_{2}}$. Furthermore by evaluating the ratios $\frac{x}{y}, \frac{x}{z}, \frac{y}{z}$ and with the constraint $c_{0}^{2}+c_{1}^{2}+c_{2}^{2}=1$, one can completely determine the values of $c_{0}, c_{1}, c_{2}$.

The next step is to judge whether the point corresponds to the maximally extremal point, where $f$ has to satisfy the relation $\frac{\partial^{2} f}{\partial a_{\alpha} \partial a_{\beta}} \leq 0(\alpha, \beta=1,2,3)$. With Eqs. (A6), one can check out easily $\frac{\partial^{2} f}{\partial a_{\alpha} \partial a_{\beta}} \leq 0(\alpha \neq \beta)$. Thus one has

$$
\left\{\begin{array}{l}
\frac{\partial^{2} f}{\partial a_{1}^{2}}=\frac{\sqrt{\left(1-a_{2}\right)\left(1-a_{3}\right)}}{4\left(1-a_{1}\right)^{3 / 2}} c_{0} \sqrt{\frac{1-a_{1}}{a_{1}}}\left(2-\frac{1}{a_{1}}\right), \\
\frac{\partial^{2} f}{\partial a_{2}^{2}}=\frac{\sqrt{\left(1-a_{1}\right)\left(1-a_{3}\right)}}{4\left(1-a_{2}\right)^{3 / 2}} c_{1} \sqrt{\frac{1-a_{2}}{a_{2}}}\left(2-\frac{1}{a_{2}}\right), \\
\frac{\partial^{2} f}{\partial a_{3}^{2}}=\frac{\sqrt{\left(1-a_{1}\right)\left(1-a_{2}\right)}}{4\left(1-a_{3}\right)^{3 / 2}} c_{2} \sqrt{\frac{1-a_{3}}{a_{3}}}\left(2-\frac{1}{a_{3}}\right) .
\end{array}\right.
$$

where Eq. (A6) is used. It is obvious that only if $a_{\alpha}<\frac{1}{2}$, then $\frac{\partial^{2} f}{\partial a_{\alpha}^{2}} \leq 0$. With this condition one can easily find the maximally extremal values of $f$.

Finally it should be pointed that the maximal values are in general not completely consistent with the extremal values. Thus the boundary points $a_{\alpha}=0,1$ must be checked independently.
[1] M. B. Plenio and S. Virmani, Quant. Inf. Comp. 7, 1 (2007); R. Horodecki, P. Horodecki, M. Horodecki and K. Horodecki, Rev. Mod. Phys. 81, 865-942 (2009).

[2] W. Dür, G. Vidal, J.I. Cirac, Phys. Rev. A 62,062314 (2000).

[3] V. Coffman, J. Kundu and W. K. Wootters, Phys.Rev.A 61, 052306 (2000).

[4] T. J. Osborn and F. Verstraete, Phys. Rev. Lett. 96, 220503 (2006).

[5] V. Vedral and M. B. Plenio, Phys.Rev. A57, 1619 (1998).

[6] A. W. Harrow and M. A. Nielson, Phys. Rev. A 68, 012308 (2003).

[7] M. Horodecki, P. Horodecki and R. Horodecki, Phys. Lett. A 223, 1 (1996); P. Horodecki, Phys. Rev. A 68, 052101 (2003).

[8] T. C. Wei and P. M. Goldbart, Phys. Rev. A 68, 042307(2003); J. Phys. A: Math. Gen. 37, 2965 (2004).

[9] H. T. Cui, Phys. Rev. A 81, 042112 (2010).

[10] A. Botero, and B. ReznikarXiv: 0708.3391v3 [quantph]; R. Orús, Phys. Rev. Lett. 100, 130502 (2008); R. Orús, Phys. Rev. A 78, 062332 (2008); Tzu-Chieh Wei,
Phys. Rev. A 81, 062313 (2010); Jean-Marie Stéphan, G. Misguich, F. Alet, arXiv:1007.4161v1 [cond-mat.statmech]; R. Orús, Tzu-Chieh Wei, Phys. Rev. B 82, 155120 (2010); Qian-Qian Shi, R. Orús, J. Ove Fjærestad and Huan-Qiang Zhou, New J. Phys. 12, 025008 (2010); R. Orús and T.-C. Wei, arXiv:1010.5029v1 [cond-mat.strel].

[11] T. C. Wei, D. Das, S. Mukhopadyay, S. Vishveshwara, and P. M. Goldbart, Phys. Rev. A 71, 060305R (2005); C.-Y. Huang and F.-L. Lin, Phys. Rev. A 81, 032304 (2010)

[12] H. T. Cui, C. M. Wang and S. Z. Yuan, Euro. J. Phys. B 74, 543 (2010).

[13] P.W. Anderson, Phys. Rev. Lett. 18, 1049(1967); G.D. Mahan, Many-Particle Physics, 3rd Edition, (Kluwer Academic/Plenum, New York, 2000).

[14] Y. Cao and A. M. Wang, J. Phys. A: math. and Theo. 40, 3507 (2007).

[15] R. Orús, S. Dusuel, and J. Vidal, Phys. Rev. Lett. 101, 025701 (2008).

[16] T. Z. Wei and S. Severini, J. Math. Phys. 51, 092203 
(2010).

[17] R. Hübener, M. Kleinmann, T. C. Wei, O. Gühne, Phys. Rev. A 80, 032324 (2009).

[18] M. Hayashi, D. Markham, M. Murao, M. Owari and S. Virmani, J. Math. Phys. 50, 022104 (2009).

[19] S. Tamaryan, A. Sudbery and L. Tamaryan, Phys. Rev. A 81, 052319 (2010).

[20] J. Martin, O. Giraud, P. A. Braun, D. Braun, T. Bastin, Phys. Rev. A 81, 062347 (2010).

[21] R. Orús and T. Z. Wei, arXiv: 1006.5584[cond-mat].

[22] W. Son, L. Amico, S. Pascazio, R. Fazio and V. Vedral, arXiv: 1001.2656[quant-ph].

[23] The meaning of the next-nearest-neighbor site in this article is defined by the sequence of all party labels with geometry shown in Fig. 1

[24] N. Li and S. Luo, Phys. Rev. A 78, 024303 (2008); K. Modi, T. Paterek, W. Son, V. Vedral, and M. Williamson, Phys. Rev. Lett. 104, 080501 (2010).

[25] The geometry of circle automatically implies the periodic boundary condition for multipartite state, as shown in Fig 1
[26] As for $|\bar{W}\rangle_{4}=\frac{1}{2}(|0111\rangle+|1011\rangle+|1101\rangle+|1110\rangle)$, it is obvious that $|\bar{W}\rangle_{4}=\otimes_{n=1}^{4} \sigma_{n}^{x}|W\rangle$. Thus $|\bar{W}\rangle_{4}$ is equivalent to $|W\rangle$, and in addtion $|\bar{W}\rangle_{4}$ has also the same TI to $|W\rangle$ because $\sigma_{n}^{x}$ operates every party at the same time. Thus it is not necessary to discuss this case solely. This principle is also applied in the following discussion; If two entangled states could be transformed into each other by the local unitary operation, which is the tensor product of the same one unitary singl-party operator, e.g. $\otimes_{n=1}^{4} \sigma_{n}^{x}$ for the four-qubit case, only one of them will be discussed in this paper. It should be pointed out that $\left|\mathrm{GHZ}^{\prime}\right\rangle_{N}$ thus has a different TI from $|\mathrm{GHZ}\rangle_{N}$ because the operation $\sigma_{2}^{x} \otimes \sigma_{4}^{x} \otimes \cdots \otimes \sigma_{2 n}^{x} \otimes \cdots$ is only imposed on the states in even site.

[27] This point becomes clearer for the case including $|W\rangle_{N}$ because $|W\rangle_{N}$ has the same common coefficient $1 / \sqrt{N}$ to that of basic $N$-period TI entangled states, which excludes the effect of the superposition coefficients.

[28] H. A. Carteret, A. Higuchi and A. Sudbery, J. Math. Phys. 41, 7932-7939 (2000). 